Astrophysical Journal, 536, June 10, 2000

\title{
DYNAMICS OF WARM ABSORBING GAS IN SEYFERT GALAXIES: NGC 5548
}

\author{
Mark C. Bottorff \\ Department of Physics \& Astronomy, University of Kentucky, Lexington, KY 40506-0055 \\ Kirk T. Korista \\ Department of Physics, Western Michigan University, Kalamazoo, MI 49008 \\ and \\ Isaac Shlosman \\ Department of Physics \& Astronomy, University of Kentucky, Lexington, KY 40506-0055
}

\begin{abstract}
A hydromagnetic (MHD) wind from a clumpy molecular accretion disk is invoked to explain observations of warm absorbing gas at UV and X-ray energies in Seyfert 1 galaxies. This paper focuses on two important issues: (1) compatibility of kinematics and dynamics of an MHD wind with the observed properties of warm absorbers; and (2) the relationship between the UV and X-ray absorbing gases. We provide an in-depth comparison between the MHD wind model and the well-studied Seyfert 1 galaxy NGC 5548, which at high spectral resolution exhibits a number of discrete UV absorption components. Model parameters used in this study have been fixed by our previous work on the variability of broad-line region in this object. The detailed UVX absorption structure in NGC 5548 is modeled in order to infer the position, size, density and temperature of each component, as well as the overall dynamics of the absorbing gas.

We find that for NGC 5548: (1) the total column densities of O VII, O VIII and H, inferred from X-ray observations are reproduced by constraining the UV ion column densities of $\mathrm{C}$ IV and $\mathrm{N} \mathrm{V}$ in each component to lie within a factor of 2 of their observed values and optimizing over the possible sets of component ionization states and C IV column densities; (2) the warm absorbing gas exists in the outer part of the wind and is not a continuation of the flow in the broad emission-line region; and (3) the warm absorber extends both in radial and polar directions and is ionization stratified. X-ray absorption is found to be heavily biased towards smaller radii and UV absorption originates at larger distances from the central continuum source. Our analysis shows that the discrete absorption components along the line-of-sight are intrinsically clumpy. Density differences between kinematic components result in a range of ionization and recombination time scales.

We further test the applicability of the MHD wind to warm absorbers in general, by constructing a quasi-continuous flow model, and extending it to arbitrary aspect angles. Constraining the ionization and volume filling factor for a generic case, we estimate the fraction of Seyfert 1s having detectable warm absorbers with larger O VII column density than $\mathrm{O}$ VIII, and the range of total hydrogen column densities. We also find that the ratio of $\mathrm{O}$ VII to $\mathrm{O}$ VIII optical depths can serve as a new diagnostic of AGN aspect angle.

Finally, the thermal stability of the UVX absorption model is tested. We find that all kinematic components in NGC 5548 are thermally stable to isobaric perturbations. In a more general case, we show that the magnetic field is crucial in order to stabilize the warm absorber gas over a wide range of incident continuum spectral energy distribution and gas metallicity.
\end{abstract}


Subject headings: galaxies: active - galaxies: individual (NGC 5548) galaxies: nuclei — galaxies: Seyfert — galaxies: ultraviolet — galaxies: X-rays 


\section{INTRODUCTION}

Highly-ionized, tenuous, absorbing gas has been detected in the soft X-ray spectra of many active galactic nuclei (AGNs) at low and moderate $(\Delta E / E=$ $0.02-0.1)$ spectral resolutions. At least half of the Seyfert 1 galaxies observed by the $A S C A$ satellite indicate the presence of this "warm absorber" gas (hereafter WA) in their X-ray spectra (e.g., review by Mushotzky 1997). Other AGNs, such as radio-quiet and radio-loud quasars, host WA as well (e.g., Green and Mathur 1996, Mathur and Elvis 1995, Siebert et al. 1996, Ulrich et al. 1999), though no statistics are currently available. In addition, broad absorptionline quasars (hereafter BAL QSOs), which constitute at least $10 \%$ of all quasars, are conspicuously weak in the soft X-rays - a possible signature of a WA gas (Mathur, Elvis, \& Singh 1995; Green \& Mathur 1996; Gallagher et al. 1999).

The X-ray WA gas is inferred from the softening of the continuum at the low X-ray energies, between $0.6 \mathrm{keV}$ and a few $\mathrm{keV}$, and is due to many unresolved absorption edges from highly ionized species of elements, such as $\mathrm{O}, \mathrm{Ne}$, etc. The strongest edge depths correspond to $\mathrm{O}$ VII and $\mathrm{O}$ VIII at 0.72 and $0.85 \mathrm{keV}$, respectively. It is generally found that the total hydrogen column density of absorbing material lies between $10^{21} \mathrm{~cm}^{-2}$ and $10^{23} \mathrm{~cm}^{-2}$, and may even reach $10^{24} \mathrm{~cm}^{-2}$ (Reynolds et al. 1997, hereafter R97; George et al. 1998, hereafter G98). The energy resolution of the $A S C A$ satellite data does not allow for a study of the kinematics of the WA gas based on the X-ray absorption profiles. The thermodynamic state of the gas, however, including its temperature and density, can be inferred with a moderate amount of modeling and invoking observed X-ray variability (Otani et al. 1995, Reynolds \& Fabian 1995; R97).

Intrinsic blueshifted UV absorption was detected in AGNs by the IUE (Bromage et al. 1985; Koratkar et al. 1996; Leech et al. 1991; Walter et al. 1990; Voit et al. 1987; Verón et al. 1985) and HUT (e.g., Kriss et al. 1992). High and sometimes low-ionization lines are observed, e.g., C IV, N v, O vI, Si IV, Mg II, and others. The higher $\mathrm{S} / \mathrm{N}$ and spectral resolution data taken by the HST found many more cases of blueshifted intrinsic absorption in the UV resonance lines. Preliminary statistics indicate that intrinsic UV absorption is present in at least half of Seyfert 1 galaxy spectra (Crenshaw et al. 1999 [C99]). In addition to the BAL QSOs, there are some quasars that exhibit intrinsic narrow absorption features (see, for example, Mathur, Wilkes, \& Elvis 1999).

In all known cases, the UV resonance line absorption is present in sources with known soft Xray absorption, hinting of a connection between the two types of absorption (C99; Mathur et al. 1999; Brandt, Laor, \& Wills 1999), though the nature of this connection is not yet clear. The correspondence between the UV and X-ray absorbing gas was advanced by Mathur et al. (1995), on the basis of single slab modeling of UVX absorbing gas in NGC 5548, using HST/FOS, ROSAT, and ASCA data in their analysis. Similar analysis was performed for other objects (e.g., NGC 3516, Mathur et al. 1997; NGC 3783, Shields \& Hamann 1997). The mean FOS spectrum of NGC 5548 represented the best UV data at the time, and its low spectral resolution $\left(\sim 200 \mathrm{~km} \mathrm{~s}^{-1}\right)$ justified the single slab approximation. Much higher spectral resolution HST GHRS and STIS/Echelle have since revealed the presence of a number of distinct kinematic components within the outflowing material, with FWHM of $50 \mathrm{~km} \mathrm{~s}^{-1}-160 \mathrm{~km} \mathrm{~s}^{-1}$ (Crenshaw \& Kraemer 1999). These high resolution spectra of NGC 5548 have uncovered at least five separate C IV absorption components (C99; Crenshaw \& Kraemer 1999; Mathur, Elvis, \& Wilkes 1999 [MEW99]).

The presence of a structure within the intrinsic absorption adds another degree of complexity to the kinematical models of AGNs. It is important, therefore, to understand the relationship between the outflowing highly-ionized gas, the broad emission-line (BEL) and absorption-line regions in these objects. Numerous models have been proposed to address this problem (e.g., Blandford 1990; Arav, Shlosman \& Weymann 1997; Königl \& Kartje 1994). In this paper we extend the hydromagnetic wind model of Emmering, Blandford and Shlosman (1992, hereafter EBS), which provides a dynamical explanation for the the properties of the broad emission-line regions (BLRs) in AGNs (Bottorff et al. 1997, hereafter Paper 1), to 
the UVX absorption phenomena. In the EBS model, gas in a dusty molecular accretion disk is loaded on magnetic field lines and centrifugally launched forming a magnetized wind. The basic difference between the EBS model and other outflow models is that it provides for a $3 \mathrm{D}$ wind dynamics, with helical motion around the axis being superposed onto the radial motion. The wind is clumpy and stratified both in density and ionization. Paper 1 addressed the radial wind structure at smaller latitudes above the optically-thick disk. First, it was found that the BLR is geometrically thick and has substantial optical depth. As a result, the inner part of the wind becomes progressively ionized with increasing latitude, while the outer part of the wind remains cold and molecular as long as it is shielded by the BLR. As the outer material continues to rise it becomes ionized and eventually passes the observer's line-of-sight, where the wind is detected via absorption in both UV and X-ray energy bands. The line-of-sight velocity of the wind depends on its injection radius on the disk, so a range of velocities is expected. The hydromagnetic wind model, therefore, naturally explains both the UV emission and absorption systems seen in C IV and other lines, as well as X-ray absorption. While our approach is general, we emphasize the results within the context of observations of NGC 5548 .

In Section 2, we summarize the important observations of the WA gas, including the column densities of $\mathrm{O}$ VII, $\mathrm{O}$ VIII and total $\mathrm{H}$ from the X-ray data, and the column densities of $\mathrm{C}$ IV, N V and $\mathrm{H}$ I from the UV data. In Section 3, we consider specifically the case of NGC 5548 and its discrete UV absorption components. The velocity field of the EBS model adopted in Paper 1 is then used to determine the location and line-of-sight width of the UV absorption components. The observed column densities of $\mathrm{N} \mathrm{V}$ and C IV in conjunction with the location and line-ofsight width of each component allow us to determine the density, temperature, volume filling fraction and column densities of $\mathrm{O}$ VII, $\mathrm{O}$ VIII, $\mathrm{H}$ I and total $\mathrm{H}$ via photoionization modeling. The calculated ionic column densities are compared with observations, to determine whether or not the X-ray absorption in NGC 5548 is compatible with the model and in or- der to determine the association between the UV and X-ray warm absorbers.

Section 4 describes a generic WA model. The multicomponent absorption, as seen in the C IV UV absorber of NGC 5548, is smoothed into an equivalent single absorbing column, to study the effects of a distributed WA in Seyfert galaxies without specific knowledge of kinematic absorption components. The generic model is investigated for arbitrary aspect angles to the observers. We estimate the fraction of AGNs having detectable WA gas, and larger O VIII than O VII optical depth. Finally, in the Appendix, we discuss the general thermal stability of magnetized UVX absorbing gas.

\section{OBSERVATIONAL SUMMARY}

The spectral resolution of $A S C A$ is insufficient to clearly resolve and measure oxygen and other element absorption edges without additional modeling. This is achieved by producing simplified photoionization models of WA gas using observed AGN continua. Free parameters in the model are subsequently tuned to obtain the best fit for the calculated transmitted spectrum to the observed one.

A common approach taken by R97 and G98 makes a number of essential assumptions, namely, the WA is considered (1) to be a thin slab; (2) to have a constant total hydrogen number density; (3) to be in thermal equilibrium; (4) to be exposed to a powerlaw soft X-ray continuum of photon index $\Gamma \sim 2$, and (5) to possess solar abundances. The total hydrogen column density (or equivalently slab thickness) $N_{H}$, the X-ray ionization parameter $U_{X}$ and $\Gamma$ are then varied until an optimal fit to the data is obtained. Additional free parameters include Galactic absorption, a fraction of unattenuated central continuum, a covering factor of the emitting gas, etc.

The main inferred properties of WA gas from Xray observations are the optical depths of O VII and $\mathrm{O}$ viII, and the column density of total $\mathrm{H}$ along the line-of-sight. Ionization stratification in WA gas due to differences in density and location is indicated in 
at least one object, namely MCG 6-30-15. The latter exhibits variability in the $\mathrm{O}$ VIII edge depth which is anticorrelated with the continuum, and little or no variability in O VII (Fabian et al. 1994, 1995; Otani et al. 1995). The difference in response is frequently attributed in literature to the recombination time scale, $t\left(X_{i}\right)$, of ion $X_{i}$ given by

$$
t\left(X_{i}\right)=1 / \alpha\left(X_{i}, T_{e}\right) n_{e},
$$

where $n_{e}$ is the electron density, and $\alpha\left(X_{i}, T_{e}\right)$ is the recombination coefficient, which is a function of the ion species $X_{i}$ and a slowly varying function of electron temperature $T_{e}$ (but see section 3.3.5). In such a case, over a wide range in temperature $\alpha\left(O\right.$ VII, $\left.T_{e}\right)$ and $\alpha\left(O \mathrm{VIII}, T_{e}\right)$ are of the same order of magnitude, so recombination time differences between the two ions are mainly due to differences in density. The response of $\mathrm{O}$ VIII and the lack of response of $\mathrm{O}$ VII in MCG 6-30-15 during the observing time is interpreted as evidence that $\mathrm{O}$ VII absorption occurs in a lower density gas than the O VIII absorption. Photoionization modeling and absorption depth variability in MCG 6-30-15 also indicate that the O VII and O VIII absorption occurs at different locations (Otani et al. 1995). An additional indication of ionization stratification in WA AGN gas is that objects with significant optical reddening exhibit largest $\mathrm{O}$ VII absorption a hint that X-ray absorption may occur in regions where dust can survive (Komossa \& Fink 1997).

If the UV and X-ray absorbers are related, then some of the assumptions used in previous modeling efforts must be modified in view of the detection of multiple kinematic components in Seyfert galaxies, in particular in NGC 5548, which exhibits 5 separate, blueshifted UV absorption components. Thus, we abandon the single zone model in favor of a multiple zone constrained by the EBS model. The constant density approximation is relaxed by estimating density for each of the kinematic components separately, by placing them at different distances from the central continuum source, based on kinematical constraints of the EBS model. We assume thermal and ionization equilibrium in the UVX gas (but see Krolik \& Kriss 1995, and Nicastro et al. 1999). Target values for the total hydrogen column density, $N_{H}$, and the optical depths $\tau(O$ VII $)$ and $\tau(O$ VIII $)$, which we convert to column densities, are adopted from the $\mathrm{X}$-ray observations discussed in R97 and are listed in Table 1a.

In addition to the observations at X-ray energies, we also consider the HST UV GHRS observations of the 5 discrete, blueshifted absorption components of C IV, N V and H I detected in NGC 5548 on 1996 Aug. 24 and on 1996 Feb. 17 (C99) (see Table 1b). We adopt the ionic column densities measured from the GHRS observations over the STIS observations (Crenshaw \& Kraemer 1999) because the former are closer in time to the $A S C A$ X-ray observations of NGC 5548 on 1993 July 27 (R97). The single exception is for the column density of $H$ I which is lacking in component 1 in the GHRS spectra so we adopt the value from the STIS data. Note that the UV observations are separated by seven months and the X-ray observations are separated from the UV observations by about 2 years. Extensive multiwavelength monitoring campaigns of this object (Clavel et al. 1991; Korista et al. 1995) show variations of UV emission lines which correlate with the continuum over times of days to weeks. C IV emission in NGC 5548 has, for example, varied in strength by a factor of $\sim 2$ in a month's time and C IV absorption equivalent width may be similarly variable (Shull \& Sachs 1993). Caution must therefore be taken when using noncontemporaneous data to determine the ionization state of the absorbing gas. We also point out that reported values of the ionic column density depend upon the procedure used to fit absorption line profiles. For example, given the same set of $H S T /$ GHRS spectra of the C IV absorption systems, MEW99 and C99 derived values of the C IV column densities of components $1-3$ which differed by $0.2-0.5$ dex. Next, modeling that goes into determining observationallyderived ionic column densities does not explicitly account for the possible effects of continuum scattering into the absorption troughs, although these effects do appear grossly in the estimate of the incomplete line-of-sight continuum coverage. This modeling must also make assumptions concerning the underlying emission from the narrow and BEL regions. Finally, the absorption lines are often observed to be 
saturated, yet non-black. The line-of-sight covering fraction of the ions along the various line-of-sight velocities must be estimated from the degree of saturation present. This is usually done by comparing the depths of the resonance line doublet pairs. Differences in line-of-sight effective continuum coverage amongst the various ions can be expected and are often found. The H I column densities reported by C99 and Crenshaw \& Kraemer (1999) suffer from a lack of constraints in this respect (Ly $\alpha$ is a singlet, and no higher order Lyman line data are available), and should therefore be considered as lower bounds (Crenshaw, private communication). Thus the uncertainties in the ionic column densities reported in the literature are internal uncertainties only, the total uncertainties must be significantly larger.

Our adopted ionic column density set is listed in Tables 1a and 1b. Given the above considerations, we have disregarded their reported uncertainties, and instead assigned a factor of 2 uncertainty above and below their reported values. An exception is component 1 of $\mathrm{C} \mathrm{IV}$, which at the limit of detection is treated as a lower bound (Crenshaw, private communication). These assigned ranges of uncertainty about the reported ionic column density values, which we will refer to as observational constraints, will be used to find a plausible set of solutions to the UVX absorber in NGC 5548.

\section{DYNAMICS AND EMISSION MODELING FOR AGNs}

\subsection{Hydromagnetic Wind Model}

The magnetohydrodynamic (MHD) solution for a stationary, axisymmetric flow in cylindrical coordinates is given by Blandford \& Payne (1982) and describes a self-similar, cold, nonrelativistic MHD flow from an idealized Keplerian disk around a point mass. This solution can be written in terms of variables $\chi$, $\xi, \phi$ and a scaling parameter $r_{0}$ which are related to cylindrical coordinates via $\mathbf{r} \equiv\left[r_{0} \xi(\chi), \phi, r_{0} \chi\right]$. Here, $\chi$ is the coordinate along a field line, $\xi(\chi)$ is found as part of a self-consistent solution to the MHD equa- tions, and $r_{0}$ is the field line footpoint on the disk. The flow velocity components are given by

$$
\mathbf{v}=\left[\xi^{\prime}(\chi) f(\chi), g(\chi), f(\chi)\right] \sqrt{G M / r_{0}},
$$

where a prime denotes differentiation with respect to $\chi$, and $M$ is the mass of the central black hole. The EBS provide for a non-trivial extension of Blandford \& Payne solution for an arbitrary scaling of volume density $n \propto r_{0}^{-b}$ and magnetic field $B \propto r_{0}^{-(b+1) / 2}$. At the base of the flow, the rotational velocity, $v_{\phi}$, is Keplerian so $v_{\phi} \propto r_{0}^{-1 / 2}$. The functions $\xi(\chi), f(\chi)$ and $g(\chi)$ are chosen to satisfy the flow MHD equations subject to the above scalings of $\rho, B$ and $v_{\phi}$. This implies that the Alfvén speed scales with the disk Keplerian velocity, and the specific angular momentum and energy in the flow will scale similarly to their Keplerian counterparts, while the disk mass loss per decade in radius scales as $\propto r_{0}^{-(b-1.5)}$. The above velocity law is of course a non-Keplerian one at all finite distances from the disk (e.g., EBS and Paper 1).

EBS utilized an analytic approximation for $\xi(\chi)$ in which $\xi(\chi) \sim \chi^{1 / 2}$, and asymptotically tends to the Blandford \& Payne solution. To attach the solution to the disk at $r_{0}, \xi(\chi)$ was constructed to have the form $\xi(\chi)=\sqrt{\chi / c_{2}+1}$, so that $\xi(0)=1$. The constant $c_{2}=\left(\tan \theta_{0}\right) / 2$, where $\theta_{0}$ is the initial angle between the magnetic line and the disk. The MHD equations have been solved for $f(\chi)$ and $g(\chi)$, so as to be consistent with the analytic form of $\xi(\chi)$. The model parameters have been fixed in Paper 1 by modeling the BLR response to the variation of the central continuum (see section 3.2). We use $b=1.5$, so $n$, the particle density, scales as $n \propto R^{-3 / 2}$ and $B \propto R^{-5 / 4}$, where $R$ is the spherical radius from the central mass. We show below that this choice in $b$ is consistent with the variation in density inferred from observations, although the number of observational points is small. This result, therefore, should be taken with necessary caution. We have further neglected the effect of radiation pressure on the gas dynamics (e.g., de Kool \& Begelman 1995), as it will not qialitatively change our results. 


\subsection{Constraining the MHD Flow with the BLR Spectrum}

With an analytical solution for the volume emissivity EBS showed that realistic BLR emission line profiles can be produced. Paper 1 continued these efforts replacing the analytic emissivity function with an emissivity function obtained from a fit to an amalgam of optically thick clouds calculated by the photoionization code Cloudy (Ferland 1996). Emission line anisotropy and finite optical depth effects were introduced to model C IV emission from optically thick BLR clouds in the well studied object, NGC 5548 . As part of the modeling, variations in C IV emission in response to observed variations of the continuum, including light travel time effects, were studied. A time series of synthetic C IV emission line profiles were generated and compared with the observed C IV emission line profiles from the HST (Korista et al. 1995), and a model fit to the data was thereby achieved. The BLR parameters in NGC 5548 deduced from the model were the central black hole mass $\left(3 \times 10^{7} M_{\odot}\right)$, physical extent of the $\mathrm{C}$ IV emitting gas (1 to 24 light days) and the orientation of the observer line-of-sight relative to the axis of symmetry $\left(40^{\circ}\right)$. Here, we extend the results of Paper 1 and analyze the flow beyond the BLR and at larger latitudes above the disk, in particular as the flow crosses the observer's line-ofsight.

In the ideal EBS flow, cold molecular gas is launchec from a Keplerian disk and is flung along the magnetic field lines, like beads along a rotating wire. Most of the magnetic lines, however, are inclined unfavorably and hence will not be able to accelerate the gas. The centrifugally accelerated gas becomes illuminated by the central ionizing continuum at some latitude. The distribution of densities and ionization parameters, combined with the MHD model's velocity field produces typically observed broad emission-line profiles. For NGC 5548, Paper 1 found that the BLR lies within a toroidal wedge $\pm 30^{\circ}$ of the equatorial disk plane. The boundary of the wedge was interpreted as coming from optically-thick wind filaments experiencing thick-to-thin transition. In this section, we focus on the optically thin (to the ionizing continuum) flow, which is the continuation of the BLR flow, as it crosses the line-of-sight to the observer. Due to rather unfavorable conditions for loading and acceleration at the base of the wind, it is natural to expect a number of separate kinematic components along the line-ofsight. The five blueshifted UV absorbers reported in C99 and MEW99 for NGC 5548, while perhaps not fully distinct from one another, are interpreted here as kinematically distinct regions containing gas in an EBS-type magnetized wind. Moreover, each individual component is expected itself to be clumpy and is characterized by a volume filling factor, as we show below.

A point on a streamline is defined in terms of the footpoint radius $r_{0}$, the spherical radius $R$, and the observer's aspect angle $i$ with respect to the disk axis, namely

$$
r_{0}=Q\left(i, \theta_{0}\right) R
$$

where

$$
Q\left(i, \theta_{0}\right)=\frac{\sqrt{\cos ^{2} i+\tan ^{2} \theta_{0} \sin ^{2} i}-\cos i}{\tan \theta_{0}} .
$$

In Paper 1, for the best fit model of the BLR in NGC 5548, the launch angle $\theta_{0}$ and aspect angle $i$, were found to be $20^{\circ}$ and $40^{\circ}$, respectively. In this case, $R \approx 10.4 r_{0}$. Figure 1 illustrates the model geometry for NGC 5548. A patch of gas filaments lifts off the disk between cylindrical radii $r_{1}$ and $r_{2}$ and rises along a helical trajectory, forming a parabola in the $r z$-plane. Gas that starts at $r_{1}$ eventually crosses the observer line-of-sight at a spherical radius $R_{1}$ and gas that starts at $r_{2}$ does this at $R_{2}$.

The number density of a total hydrogen along the flow line that starts at $r_{0}$ is prescribed by the model to be

$$
n=\frac{n_{A}}{m}\left(\frac{r_{0}}{r_{1}}\right)^{-1.5} \text {. }
$$

Here $n_{A}$ is a normalization constant, setting the density $n$ on the innermost flow line at $m=1.0$, where $m$ is the Alfvén Mach number. The value of $m$ increases from unity at about $17^{\circ}$ above the disk to about $10^{3.3}$ at $85^{\circ}$ above the disk. The ionization parameter $U \propto 1 / n R^{2}$, therefore, scales as $U \propto m Q^{2}\left(i, \theta_{0}\right)$ along a flow line. At small latitudes, the effective ionization parameter for lower column density filaments 


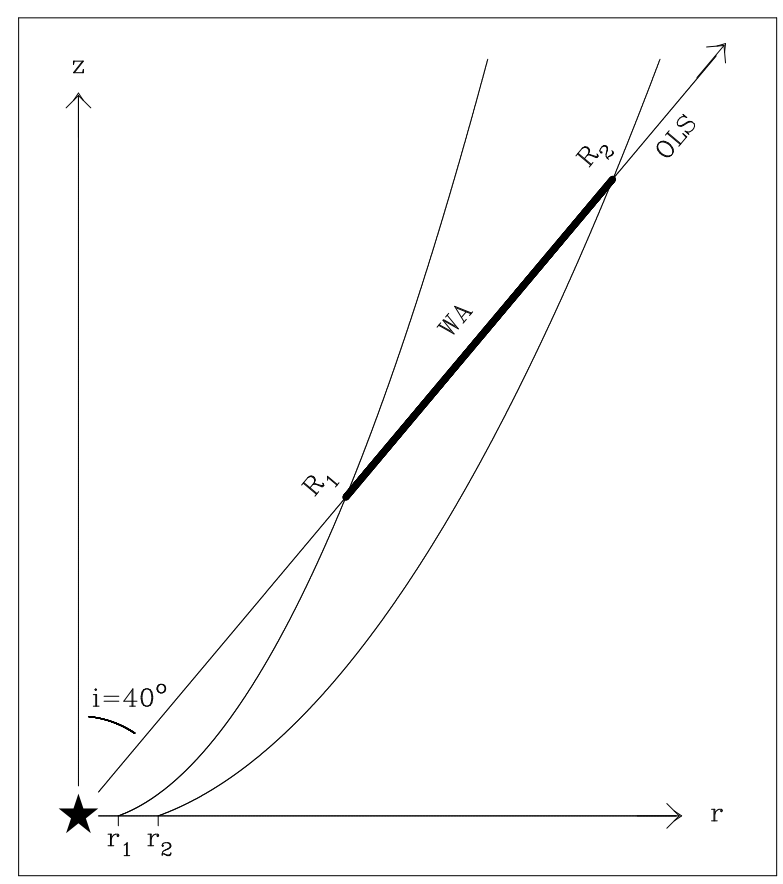

Fig. 1. - Schematic representation of the warm absorber column. Gas, destined to contribute to the warm absorber, rises off a disk in the $z=0$ plane between $r_{1}$ and $r_{2}$ and crosses the observer's line-ofsight (OLS) between $R_{1}$ and $R_{2}$, where it is detected via continuum absorption. Gas inside $R_{1}$ is overionized, so there is little contribution to the absorption column densities of $\mathrm{O}$ VII, O VIII, $\mathrm{C}$ IV or $\mathrm{N}$ V in this region. Outside $R_{2}$ the flow has been cut off by mechanisms described in the text.

will be even smaller, due to finite optical depth effects in the BLR (Paper 1). The change in radius and density causes the ionization parameter along any flow line to have a maximum at about $30^{\circ}$ above the disk, where the BLR flow becomes optically thin. Here, the BLR flow is no longer self-shielded, becomes overionized and remains so at larger latitudes, where it crosses the observer's line-of-sight. As a result, this gas does not contribute significantly to the UVX absorbing column. The gas filaments launched at larger radii than the BLR flow are less ionized and form the bulk of the UVX absorption. This absorbing gas is indicated by the thick line segment in Figure 1.

While the inner boundary of the wind is set by photoionization, the outer boundary can be fixed by considering the amplification of magnetic fields in the disk. An efficient generation of magnetic fields in the disk will cause its expulsion through the buoyancy effects and probable field reconnection above the disk (e.g., Galeev, Rosner \& Vaiana 1979). The energy release during the reconnection can in principle inject disk material and load it onto the large-scale field treading the disk, resulting in the hydromagnetic wind. While the large-scale disk-threading poloidal field is probably governed by global considerations, the disk-generated fields are expected to be amplified by differential rotation. Such dynamo effect will operate only within the "radius of influence," $r_{B H}$, of the central black hole (BH), because for $r>r_{B H}$ the inner galactic rotation is typically that of a solid body. Hence, the outer wind cutoff can plausibly result from a dramatic decrease in the shear outside $r_{B H}$, which prevents the magnetic field in the disk from reconnecting and loading the molecular material onto large-scale field lines. This radius can be estimated from equating the gravitational potentials of the $\mathrm{BH}$ and that of the host galaxy, namely, $r_{B H} \sim$ $1.3 \times 10^{19} m_{7} v_{2}^{-2} \mathrm{~cm}$, where $m_{7} \equiv M_{B H} / 10^{7} \mathrm{M}_{\odot}$ and $v_{2} \equiv v_{K} / 100 \mathrm{~km} \mathrm{~s}^{-1}$ is the rotational velocity in the galactic disk. Adopting the $\mathrm{BH}$ mass in NGC 5548 from Paper 1, leads to $R_{2} \sim 1.4 \times 10^{20} \mathrm{~cm}$ for a typical $v_{7}=2.5$. As we show below in $\S 3.3 .1$ this is within a factor of $\sim 2-3$ from the outer boundary of the observed kinematic components.

\subsection{Multi-Component Absorption Model for NGC 5548}

\subsubsection{Location of Absorption Components}

The positions of separate absorption components are calculated using the relationship between $R$ and $v_{o b s}$, the line-of-sight component of wind velocity. The velocity $v_{o b s}$ is obtained by projecting the velocity field onto the observer line-of-sight and is given by

$$
v_{o b s}=\left[\xi^{\prime}(\chi) f(\chi) \sin i+f(\chi) \cos i\right] \sqrt{G M / r_{0}} .
$$


Substituting $r_{0}=Q\left(40^{\circ}, 20^{\circ}\right) R$ and $M=3 \times 10^{7} \mathrm{M}_{\odot}$ for NGC 5548 (Paper 1), gives

$$
R=7.3 \times 10^{18}\left(\frac{v_{o b s}}{10^{3} \mathrm{kms}^{-1}}\right)^{-2} \mathrm{~cm}
$$

Observed component velocities in the rest frame of NGC 5548 from C99 and the FWHM of each component which we use below are listed in Table 3b. Inserting the observed component velocities into Equation 7 , yield component distances which range from about 2 to about 87 parsecs (Table $3 \mathrm{~b}$ ). The most important consequence is that the UVX gas in NGC 5548 appears to be positioned well outside its BLR, both in radii and in latitude. Hence, within the framework of the EBS model, which neglects the effects of radiation pressure, the the UVX absorbing gas originates in the outer part of the wind, beyond the BLR flow.

\subsubsection{Calculation of Possible Ionization States}

The observational constraints on the ionic column densities and their ratios in section 2 are now used to find the limits on the ratios of the $\mathrm{N} \mathrm{V}$ ion fraction, $f(N \mathrm{~V})$, with the $\mathrm{C}$ IV ion fraction, $f(C \mathrm{IV})$. These estimates allow the determination of a set of possible gas ionization states, i.e., the particle density, electron temperature and various ion fractions in each of the UVX absorption components. We assume a total hydrogen number density $n$ as given by equation 5 , so the column density $N\left(X_{i}\right)$ of ion $X_{i}$ is

$$
N\left(X_{i}\right)=\int_{\Delta R} \epsilon f\left(X_{i}\right) a_{X} n d R \approx \epsilon f\left(X_{i}\right) a_{X} \int_{\Delta R} n d R,
$$

where $\epsilon$ is the volume filling factor in the gas column which covers the central continuum source, $f\left(X_{i}\right)$ is the ion fraction, $a_{X}$ is the abundance of element $X$, and $\Delta R$ is the line-of-sight geometrical width of the cloud. If the ionization parameter is a slowly varying function of radius with little change over $\Delta R$ and $\epsilon$ is constant within each component, then $\epsilon f\left(X_{i}\right) a_{X}$ can be taken out of the integral. Then $f(N$ v $) / f(C$ IV $)$ for each component is given by

$$
\frac{N(N \mathrm{~V})}{N(C \mathrm{IV})} \approx \frac{f(N \mathrm{~V})}{f(C \mathrm{IV})} \frac{a_{N}}{a_{C}}
$$

Assuming solar abundances, $a_{N}=9.33 \times 10^{-5}$ and $a_{C}=3.55 \times 10^{-4}$, we obtain the ion fraction ratio

$$
\frac{f(N \mathrm{~V})}{f(C \mathrm{IV})} \approx 3.805 \frac{N(N \mathrm{~V})}{N(C \mathrm{IV})}
$$

The central values of $\log [f(N \mathrm{v}) / f(C$ IV $)]$ for components $1-5$, assuming the column densities listed in C99 and solar abundances, are listed in the final column of Table 1. In accord with section 2, we assign factors of two uncertainty to their values. Component 1 is a special case because the reported values for $N(C$ IV $)$ provide a lower bound only.

The range of possible ionization states for each component was determined using Cloudy (version 90.04). The luminosity and spectral energy distribution (SED) of the continuum source in NGC 5548, combined with the distance of each component (Eq. 7), is used to calculate the incident flux $\Phi(H)$ of hydrogen ionizing photons falling on each absorption component. We used the following continuum shape for the SED of NGC 5548, i.e.,

$$
f_{\nu} \propto \nu^{\alpha_{U V}} e^{-h \nu / k T_{B B}} e^{-k T_{I R} / h \nu}+a \nu^{\alpha_{X}}
$$

and

$$
\frac{f_{\nu}(2 \mathrm{keV})}{f_{\nu}(2500 \AA)}=403.3^{\alpha_{O X}},
$$

where $k T_{I R}=0.01$ Ryd is the spectrum cutoff in the near-infrared, and $a$ in Equation 11 is chosen to satisfy Equation 12. The input parameters are $\log \left(T_{B B}\right)=6.683, \alpha_{O X}=-1.20, \alpha_{U V}=-1.20$ and $\alpha_{X}=-0.90$, chosen to be in accord with IUE/ROSAT observations of the NGC 5548 continuum (Walter et al. 1994). Using the above SED, the ionizing luminosity of the continuum source is estimated at $10^{44.3} \mathrm{erg} \mathrm{s}^{-1}$ $\left(H_{0}=75 \mathrm{~km} \mathrm{~s}^{-1} \mathrm{Mpc}^{-1}\right)$, based upon the mean continuum flux at $1350 \AA$, obtained during the 1993 observing campaign (Korista et al. 1995).

With the above assumptions, the ionization parameter $U(\equiv \Phi[H] / n c)$, which controls the ionization state of the gas, is tuned by adjusting the hydrogen number density $n$ in a series of Cloudy calculations for an optically-thin slab of width $10^{10} \mathrm{~cm}$.

\footnotetext{
${ }^{1}$ Outside the range $1.36 \mathrm{eV}-100 \mathrm{keV}, a=0$. Above $100 \mathrm{keV}$, we assume $f_{\nu} \propto \nu^{-3}$.
} 
In this manner, a set of synthetic photoionization gas states covering a range of possible $f(N \mathrm{v}) / f(C$ IV $)$ values is produced for each component.

In order to provide for a complete description of the UVX absorption in each kinematic component, it is necessary to know the column densities of different ions. Note that, if we replace $N(N \mathrm{v})$ with $N\left(X_{i}\right)$ and $a_{N}$ with $a_{X}$ in Equation 9, we obtain a generic expression for the column density of ion $X_{i}$. In the approximation of Equation 9, the quantity $\left[f\left(X_{i}\right) / f(C\right.$ IV $\left.)\right]\left(a_{X} / a_{C}\right)$ is constant, so $N\left(X_{i}\right) \propto$ $N(C$ IV $)$. Thus for fixed $f(N$ v $) / f(C$ IV $)$, all ionic column densities scale directly with the $\mathrm{C}$ IV column density. The parameters $f(N \mathrm{v}) / f(C$ IV $)$ and $N(C$ IV $)$ then map out the set of possible ionic column densities for each component. We use these two parameters to determine the electron temperature $T_{e}$ and column densities of $\mathrm{N}$ v, O VI, O vII, O viII, H I and $\mathrm{H}$, on a grid of $[\mathrm{f}(\mathrm{N} \mathrm{v}) / \mathrm{f}(\mathrm{C}$ IV $), N(C$ IV $)]$ pairs that are consistent with the observational constraints.

The grid of solutions constrained by $\mathrm{f}(\mathrm{N} v) / \mathrm{f}(\mathrm{C}$ IV $)$ and $\mathrm{N}(\mathrm{C}$ IV $)$ were used to determine the range of possible values of the column densities of O VI, O VII, and $\mathrm{O}$ VIII in each component. The factor of 2 uncertainty in the observational constraints on the C IV and $\mathrm{N}$ V column densities and their ratios translates into column density uncertainties of a factor of $\sim 10$ on O VI, a factor of $\sim 100$ on O VII and a factor of $\sim 1,000$ on $\mathrm{O}$ VIII. The inferred ranges on the column densities of $\mathrm{H}$ and $\mathrm{H}$ I are no better. $\mathrm{H}$ I ranges over a factor of $\sim 100$ and $\mathrm{H}$ over a factor of $\sim 1,000$ (even more in component 1). We, therefore, obtain an optimal solution for UVX absorption in NGC 5548, within the five component sets of possible solutions. Since individual column densities of C IV and N V for each component are observed, but only total column densities are available for O VII and O VIII, we search for a solution that minimizes the chi-squares, $\chi_{O}^{2}$, for oxygen ion column densities, namely

$$
\begin{gathered}
\chi_{O}^{2}=\frac{\left(\sum_{j=1}^{5} N(O \mathrm{VII})_{j}-N(O \mathrm{VII})_{o b s}\right)^{2}}{\sigma_{O \mathrm{VII}}^{2}} \\
+\frac{\left(\sum_{j=1}^{5} N(O \mathrm{VIII})_{j}-N(O \mathrm{VIII})_{o b s}\right)^{2}}{\sigma_{O \mathrm{VIII}}^{2}} .
\end{gathered}
$$

Here $N(\mathrm{O} \text { VII })_{\mathrm{j}}$ and $N(\mathrm{O} \text { vIII })_{\mathrm{j}}$ are the calculated column densities of O VII and O VIII in a component $j$ (see Table 3$), N(\mathrm{O} \text { VII })_{\text {obs }}$ and $N(\mathrm{O} \text { VIII })_{\text {obs }}$ are the observed total column densities of $\mathrm{O}$ VII and $\mathrm{O}$ VIII (see Table 1), and $\sigma_{O}$ vII and $\sigma_{O}$ vIII are the adopted observational uncertainties (section 2) in the $\mathrm{O}$ VII and $\mathrm{O}$ VIII column densities. Our search of the solution grid yields optimal $\chi_{O}^{2}=0.13$. The total column density of O VII is overpredicted by $30 \%$ and the total column density of $O$ VIII is underpredicted by $9 \%$, compared to target values in Table 1a, placing both well within the observational constraints.

\subsubsection{An Optimal Solution for UVX Absorption using the EBS Model}

The model column densities of $\mathrm{C}$ IV, $\mathrm{N} \mathrm{v}, \mathrm{O}$ vI, $\mathrm{O}$ VII, O VIII, $\mathrm{H}$ I and $\mathrm{H}$ in each component and their sum over all components are listed in Table 3a. Component $\mathrm{C}$ IV and $\mathrm{N}$ V column densities automatically satisfy the observational constraints because the optimization was done on a grid of values bounded by the constraints. The component column density values for O VI, O vII, O VIII, and $\mathrm{H}$ in each component are predictions of our model. The good match between the predicted and observed total O viI, O viII, and hydrogen column densities, given present observational constraints, indicates that the soft X-ray WA and the UV resonance-line absorber are directly related. The EBS model constrains the bulk of the WA to lie within two of the kinematic "components" of the outflow, separated by about $500 \mathrm{~km} \mathrm{~s}^{-1}$ in lineof-sight velocity. While observations at the required resolution do not yet exist to test these predictions in detail, the recently launched X-ray satellite Chandra may be able to detect the $\mathrm{O}$ VII and $\mathrm{O}$ VIII absorption edges of the individual kinematic absorption components in NGC 5548, and the recently launched FUSE satellite should at least detect the presence of absorption in $\mathrm{O}$ VI.

The predicted column densities of $\mathrm{H}$ I from our optimal solution do not match well their reported values (Table 1b). The observed component column density values are factors of $4-28$ below their coun- 
terparts in the optimal solution. However, as mentioned in section 2, the observationally inferred $\mathrm{H} \mathrm{I}$ column densities are the least certain, and are lower limits to their true values, at best.

The hydrogen number density for each component in the optimal solution as well as $T_{e}, U$ and other physical values are listed in Table $3 \mathrm{~b}$. The density derived for each component using Cloudy depends on the kinematic structure of the EBS model via Equation 7 , but is independent of the parameter $b=1.5$ used to describe the scaling of the EBS density in Equation 5. It is therefore possible to test whether the assumed value of $b$ used in Paper 1 and here in this paper is appropriate.

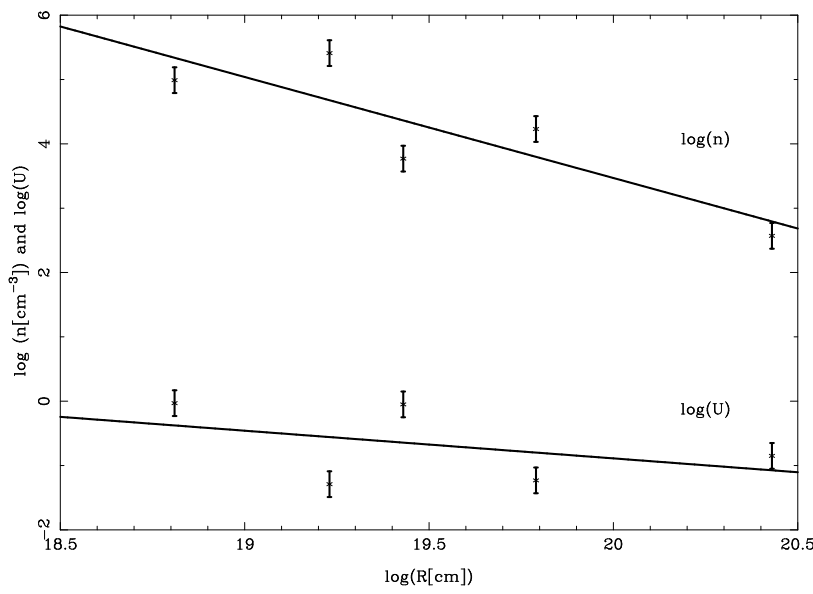

Fig. 2.- Logarithmic plot of density and ionization parameter as a function of $R[\mathrm{~cm}]$ for the discrete component model of NGC 5548. The straight lines are the least squares fits.

Equations $3-5$ together imply that along any ray through the origin $n \propto R^{-1.5}$, and, therefore, the ionization parameter varies as $U \propto R^{-0.5}$. If individual absorption components obey this density prescription, then $n$ and $U$ from component to component should vary accordingly. As a test, we fit $\log (n)$ and $\log (U)$ from the optimal solution at the center of each absorption component, as functions of $\log (R)$ with a linear least squares fit. Figure 2 shows $\log (n)$, $\log (U)$ and the least squares fit of both. The range in uncertainty is \pm 0.2 and corresponds to our photoion- ization grid's resolution in gas density. We find that the linear least squares fits to $\log (n)$ and $\log (U)$ vs. $\log (R)$ have slopes of $-1.57 \pm 0.16$ and $-0.45 \pm 0.16$, respectively. These are consistent with our specific EBS assignment of $n \propto R^{-1.5}$ and $U \propto R^{-0.5}$. Since $U$ is a slowly decreasing function of $R$, our simplification of the integral in Equation 8 is reasonable, assuming that there are no significant optical depth effects along the absorbing column and that in each component $\Delta R / R$ is sufficiently small, so that there are no significant changes in $U$ over the width of a component either. The picture that emerges for the wind model of NGC 5548 is that of optically thin filaments originating over a large range of radii in the disk, from $\sim 0.2 \mathrm{pc}$ to about $9 \mathrm{pc}$. These filaments being centrifugally accelerated along the magnetic field lines, cross the line-of-sight about ten times further out. Along the line-of-sight, the ionization parameter drops as a weak function of distance $\left(U \propto R^{-1 / 2}\right)$, but because the components are spread out over a factor of $\sim 40$ in radius, there is a factor of $\sim 6$ drop in ionization parameter so the wind is ionizationstratified along the line-of-sight. The thermal stability of kinematic components in NGC 5548 is analyzed in the Appendix.

\subsubsection{Absorption Component Widths and Volume Filling Factors}

A more complete physical picture of the UVX absorption system is provided by determining the lineof-sight width, $\Delta R$, of each absorption component. In this section we estimate the ratio $\Delta R / R$, discuss the radial gas distribution, separation of individual components, their degree of clumpiness, variation of $U$, etc.

The line-of-sight extension of each kinematic component can be estimated from the FWHM of the absorption components (given in C99) and the electron temperature, provided by the optimal solution (Table 3). The FWHM is attributed to the effects of thermal Doppler broadening and velocity gradients in the MHD flow. The component due to velocity gradients, $\Delta v$, is obtained by making a correction to 
the FWHM by subtracting the thermal broadening which we take to be $v_{s}=\sqrt{2 k T_{e} / m_{i}}$, and by taking the ion mass $m_{i}$ to be the average mass of carbon and nitrogen atoms. This average is taken because the FWHMs listed in C99 were the result of averaging the FWHM determined from the $\mathrm{N} \mathrm{V}$ and $\mathrm{C}$ IV absorption lines. Table $3 \mathrm{~b}$ lists $v_{s}$ and $\Delta v$, defined by $\Delta v=F W H M-v_{s}$. All of the $\Delta v$ values are positive, therefore, some of the line width cannot be attributed solely to thermal Doppler broadening. Equation 7 is used to determine the spatial extent $\Delta R$ of each component via

$$
\begin{gathered}
\Delta R=R\left(v_{o b s}-\frac{\Delta v}{2}\right)-R\left(v_{o b s}+\frac{\Delta v}{2}\right)= \\
4 R\left(\frac{\Delta v}{2 v_{o b s}}\right)\left(1-\left[\frac{\Delta v}{2 v_{o b s}}\right]^{2}\right)^{-2} .
\end{gathered}
$$

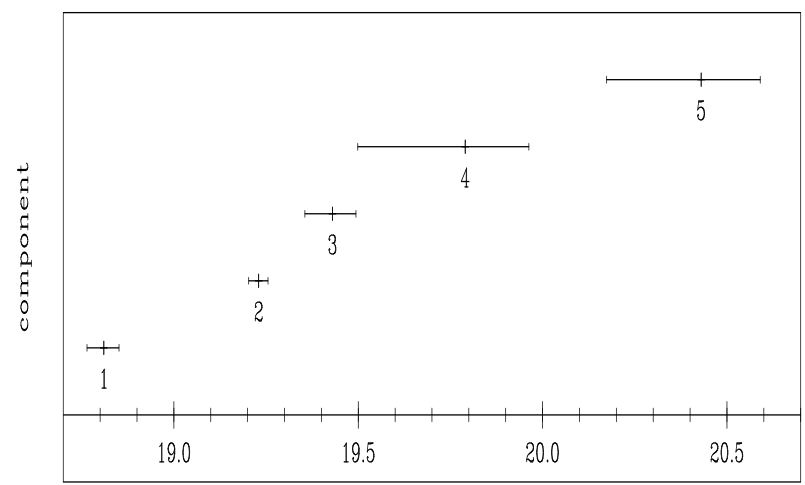

$\log (\mathrm{R}[\mathrm{cm}])$

Fig. 3.- The location and width of the discrete absorption components of the optimal model for warm absorbing gas in NGC 5548 .

Numerical values for $\Delta R$ in each component are also listed in Table 3b. Figure 3 shows the width and location of each component for the optimal solution plotted in $\log (R)$. Gaps between the components indicate that they are well separated (except for possibly components 3 and 4 ), and that $\Delta R$ values grow monotonically with $R$. For the first three components $\Delta R / R<0.32$, but it is of order unity for components 4 and 5. According to the EBS model, the ionization parameter will vary across the component by $U_{\text {front }} / U_{\text {back }}=\sqrt{(R+\Delta R / 2) /(R-\Delta R / 2)}$. The values of $R$ and $\Delta R$ for each component (Table $3 \mathrm{~b}$ ) yield percentage differences in $U$ measured at the front and back sides of each component. These are $\sim 11 \%, 6 \%, 17 \%, 71 \%$ and $61 \%$ for components 1 through 5 , respectively. Thus our approximation in Equations 8, 9 and 10 is reasonable only for the first three components. For components 4 and 5 we estimate that the effect will result in an error of about -0.1 dex in the $\mathrm{N} \mathrm{V}$ and $\mathrm{C}$ IV column densities, and as much as +0.4 dex uncertainty in the oxygen column densities. Fortunately, these errors are minor in those components where oxygen ions predominate (i.e., 1 and 3), and, therefore, the breakdown of our assumption of constant $U$ across individual components does not affect much the predicted integrated oxygen column densities.

An estimate for the volume filling factor is obtained from the column density. Using $n \propto R^{-1.5}$ as a density profile, the integral in Equation 8 is given by $\int_{\Delta R} n d R=n(R) 2 R\left[(1-\Delta R / 2 R)^{-1 / 2}-(1+\right.$ $\Delta R / 2 R)^{-1 / 2}$, where $n(R)$ and $R$ are the density and radius at the center of each component of width $\Delta R$. Hence

$$
\begin{gathered}
N\left(X_{i}\right) \approx \\
\epsilon f\left(X_{i}\right) a_{X} n(R) 2 R\left[(1-\Delta R / 2 R)^{-1 / 2}-(1+\Delta R / 2 R)^{-1 / 2}\right] .
\end{gathered}
$$

If $n\left(X_{i}\right)$ is the density of ion $X_{i}$ at $R$, then

$$
n\left(X_{i}\right)=f\left(X_{i}\right) a_{X} n(R) .
$$

Substituting Equation 16 into Equation 15, letting $q=\Delta R / 2 R$ and solving for $\epsilon$ gives

$$
\epsilon \approx \frac{N\left(X_{i}\right)}{n\left(X_{i}\right) \Delta R} \frac{q\left(1-q^{2}\right)^{1 / 2}}{(1+q)^{1 / 2}-(1-q)^{1 / 2}} .
$$

The quantity $N\left(X_{i}\right) / n\left(X_{i}\right) \Delta R$ is the filling factor, when the density is constant throughout the component. The term involving $q$ makes a correction for the variation in density across a component. The correction factors, however, are close to unity. For components 1 through 5 , they are 0.994, 0.998, 0.984, 0.844 and 0.871 , respectively. This requires the choice 
of an ion to define $\epsilon$, and we choose C IV since it is already used as one of the solution parameters. We note that $\mathrm{N} \mathrm{V}$ or even a blend of the $\mathrm{C}$ IV and $\mathrm{N} \mathrm{V}$ column densities could be used as well, but the differences in calculated $\epsilon$ are not significant.

Calculated values of $\log (\epsilon)$ for the optimal solution range from -5.58 to -1.37 (Table $3 \mathrm{~b}$ ). This means that an absorption component is clumpy and has a filamentary structure. In other words, each of the five distinct absorption components in NGC 5548 consists of filaments which move along similar trajectories and have probably a common origin in the disk. On the other hand, we presume that the distribution of points of origin of any given absorption component in the disk plane is random. The clumpiness of individual components combined with the geometry and non-radial nature of the MHD flow has an important implication for the longevity of UV absorption structures in AGNs, which we discuss in the next section.

\subsubsection{Temporal Changes in the UVX Absorbing Column}

Additional information about the character of the absorbing column of gas is inferred from the temporal behavior (or lack thereof) of UV and X-ray absorption. Changes in absorption lines in principle can be due to the motion of material into or out of the observer's line-of-sight, or it may be due to changes in the ionization structure of the gas brought about by changes in the incident continuum or both. The X-ray absorption line variability has been discussed in Reynolds \& Fabian (1995), Reynolds (1997) and Otani et al. (1995), where ASCA observations of MCG 6-30-15 show apparent anti-correlation of the $\mathrm{O}$ VIII edge depth with an increase in the continuum level. A similar conclusion was reached by Reynolds \& Fabian (1995). Observations of UV absorption lines in AGNs also reveal that they change with time. Shull \& Sachs (1993) used IUE data to show that the $\mathrm{C}$ IV absorption equivalent width in NGC 5548 responds to continuum changes in an anticorrelated fashion on a time scale less than about 4 days (this value is likely to be rather uncertain due

to the fact that the temporal sampling of this monitoring campaign was four days). UV observations of other AGNs spaced over many months reveal that absorption lines change in depth with time, but the relationship with the continuum is not clear. While the depth of an absorption line may vary, there are no unambiguous observations of a change in the velocity centroid of the line (Weymann 1997), which argues against purely radial motion, since one would expect clouds moving only radially to accelerate along the line-of-sight, resulting in time with a shift in the velocity centroid. This is in agreement with our modeling of gas dynamics in the BLR of NGC 5548 (Paper 1) using the EBS wind, which ruled out a purely radial motion as well. Here we focus on the temporal phenomena associated with UV and X-ray absorption in AGNs.

Our analysis of the discrete absorption components in the previous section suggests that each component is an agglomeration of filaments moving along the same trajectory. The heuristic model is that the wind filaments are confined by the ambient magnetic field and are extended along the field lines. The exact details of gas loading onto the magnetic lines are subject to future work. Filaments which rotate out of the line-of-sight are replace by their neighbors, so the velocity centroid of the absorption component is not expected to change with time. The time scale $t_{c}$ over which we expect an absorption structure to survive is given by $t_{c}=\Delta R / v_{\phi}$, where $\Delta R$ is the width and $v_{\phi}$ is the rotational velocity of the absorption component. For the first UV absorption component in NGC 5548 , where $t_{c}$ has the smallest value, we find $v_{\phi} \sim 10^{3} \mathrm{~km} \mathrm{~s}^{-1}$ and $\Delta R \sim 10^{19} \mathrm{~cm}$, leading to $t_{c} \sim 3,000$ years. The proposed model can, therefore, explain the observed long constancy of the velocity centroids of UV absorption structures.

Given the long dynamical time scale, observed temporal changes in the absorbing column are attributed instead to changes in the photoionization structure of the absorbing gas, rather than a change in gas density along the line-of-sight, and are presumably due to fluctuations in the incident continuum. The response of the absorption within the EBS wind to continuum fluctuations is investigated by consider- 
ing the effect of a sudden drop in the continuum level on the column density of C IV, O VII and O VIII. C IV is chosen to compare with observation (Shull \& Sachs 1993), and O VII and O VIII are chosen to compare and contrast the response of our model of NGC 5548 with observations of MCG 6-30-15.

In the above scenario, ions will begin to recombine with the drop in the continuum intensity. However, the time scale for recombination as given by Equation 1 does not account simultaneously for the cascade into the population of $X_{i}$ ions from the population of $X_{i+1}$ ions, and the cascade out of the population of $X_{i}$ ions into the population of $X_{i-1}$ ions. Equation 1 is hence misleading and is replaced for consistency by

$$
t\left(X_{i}\right)=\frac{1}{\alpha\left(X_{i}\right) n_{e}\left[\frac{f\left(X_{i+1}\right)}{f\left(X_{i}\right)}-\frac{\alpha\left(X_{i-1}\right)}{\alpha\left(X_{i}\right)}\right]} .
$$

The value of $t\left(X_{i}\right)$ in Eq. 18 establishes the minimum time, that gas will respond to a decrease in the continuum, and depends on the local density and on the ion population. As a single measure within a component, we take the average ion density-weighted recombination time which is

$$
<t\left(X_{i}\right)>=\frac{\int_{\Delta R} t\left(X_{i}\right) n_{X_{i}} \epsilon d R}{N_{X_{i}}} .
$$

This definition weights more heavily recombination times in regions with higher column density contributions. Assuming the validity of Equations $8-10$, $n_{X_{i}}, n_{X_{i+1}}$ and $n_{e}$ are all proportional to $R^{-1.5}$. In addition, $T_{e}$ within each component is approximately independent of $R$ and, therefore, $\alpha\left(X_{i}\right)$ and $\alpha\left(X_{i-1}\right)$ are also independent of $R$. The result is that $t\left(X_{i}\right) n_{X_{i}}$ is independent of $R$ and may be factored out of the integral. This gives

$$
<t\left(X_{i}\right)>=\frac{\left.t\left(X_{i}\right)\right|_{R} n_{X_{i}}(R) \epsilon \Delta R}{N_{X_{i}}},
$$

where $\left.t\left(X_{i}\right)\right|_{R}$ and $n_{X_{i}}(R)$ are $t\left(X_{i}\right)$ and $n_{X_{i}}$, respectively, evaluated at $R$. Hence, $\left\langle t\left(X_{i}\right)\right\rangle$ may be positive or negative, depending on whether or not the column density of ion $X_{i}$ is anti-correlated or correlated with a decrease in the ionizing continuum, respectively.
Calculated values of $\left\langle t\left(X_{i}\right)>\right.$ for $\mathrm{C}$ IV, O VII and O VIII in NGC 5548 are listed in Table 4. Not surprisingly, these recombination time scales differ from those calculated from Equation 1 - an issue sometimes ignored in the literature. For a fixed electron density, the ionic distributions as well as the effect of recombinations to lower ionization states can produce a wide range of the recombination time scales for the various metallic ions. In Table 4 , the computed value of $\left\langle t\left(X_{i}\right)\right\rangle$ for each ion differs from component to component. The recombination time scale for $\mathrm{C}$ IV spans a factor of $\sim 5,000$. The ranges for $\mathrm{O}$ VII and $\mathrm{O}$ VIII, span factors of $\sim 600$ and $\sim 50$, respectively. Whether or not changes of ion $X_{i}$ in a single component have a significant effect on the total ionic column density over all components of ion $X_{i}$, depends on the relative contribution of $X_{i}$ in the single component. (All reported variations in ionic UVX column densities were measured over the total value, due to insufficient spectral resolution.) For this reason the percent contributions of $\mathrm{C}$ IV, $\mathrm{O}$ VII and $\mathrm{O}$ VIII are also shown in Table 4 . The values are used to define the column density-weighted mean recombination time over all components, $<<t\left(X_{i}\right)>>$ (Table 4 ). The individual recombination times in each component serve as lower bound response time scales to changes in the ionizing continuum flux, and $<<t(C$ IV $)>>$ may be compared in a crude manner with observations. In our model, $<<t(C$ IV $)>>\approx+3.7$ days is reasonably consistent with the results of Shull \& Sachs (1993), namely, that $\mathrm{C}$ IV absorption equivalent width is anticorrelated with continuum variations with a delay of roughly four days.

Long term X-ray observations of NGC 5548 do not yet exist, so the time scales reported in Table 4 for $\mathrm{O}$ VII and $\mathrm{O}$ VIII cannot be tested. Instead we compare the results of this model to the observations of MCG 6-30-15. In our model of the UVX absorption flow in NGC 5548, the bulk of the contribution to the column density of these two oxygen ions resides in components 1 and 3 . However, the values of $<<t(O$ VII $)>>$ and $<<t(O$ VIII $)>>$ are considerably skewed by the extremely long recombination time scale of component 5 . As a conservative measure, we, therefore, consider only components 1 and 
3. In these components, the column density contributions are roughly equal for both O VII and O VIII, but the recombination times in the first component are over 10 times shorter. These recombination times, which represent the minimum possible response time scale for a continuum drop, are $\langle t(O$ VII $)>\approx-3$ days and $<t(O$ VIII $)>\approx-50$ days. (The $\mathrm{O}$ VII and $\mathrm{O}$ viII edge optical depths are correlated with the ionizing continuum intensity.) We contrast this with observations of MCG 6-30-15 (see section 2). During the second half of the $\sim 4$ day observation period of this object, the X-ray continuum dropped by a factor of 2. The response was that the O viII optical depth anti-correlated with the continuum on a time scale of $\sim 10^{4} \mathrm{~s}$, but there was no observed change in the O viI optical depth.

A possible explanation for the observed behavior of the O vIII edge optical depth in MCG 6-30-15 is that the absorbing gas in MCG 6-30-15 is more highly ionized than in NGC 5548. The observed continuum in MCG 6-30-15 is much harder than in NGC 5548, and the WA gas may, therefore, support a large population of O IX ions. Equation 18 allows then for a complex dependency of the recombination time scales, which might explain the differences between the predicted behavor of NGC 5548 and the observed behavior of MCG 6-30-15. Long looks by Chandra and other advanced X-ray telescopes should establish the relationships between the continuum and WA optical depths in AGNs.

\section{A GENERIC MODEL FOR WARM ABSORBING GAS}

The discrete UVX absorber model for NGC 5548 does not allow for its direct application to other objects. This is mainly because detailed kinematic data is not available, the model does not provide for the UVX absorption dependence on the aspect angle, and because it does not specify the appropriate boundary conditions not along the line-of-sight. To generalize the model, we proceed by spatially smoothing the contributions from individual UVX kinematic components of our model and by calculating the optical properties of the MHD flow along a number of aspect angles. The resulting generic model is used to estimate orientation effects on the soft X-ray absorption properties of AGNs. The general aspects of thermal stability of the WA gas in this model are discussed in the Appendix.

\subsection{Column Densities and physical conditions}
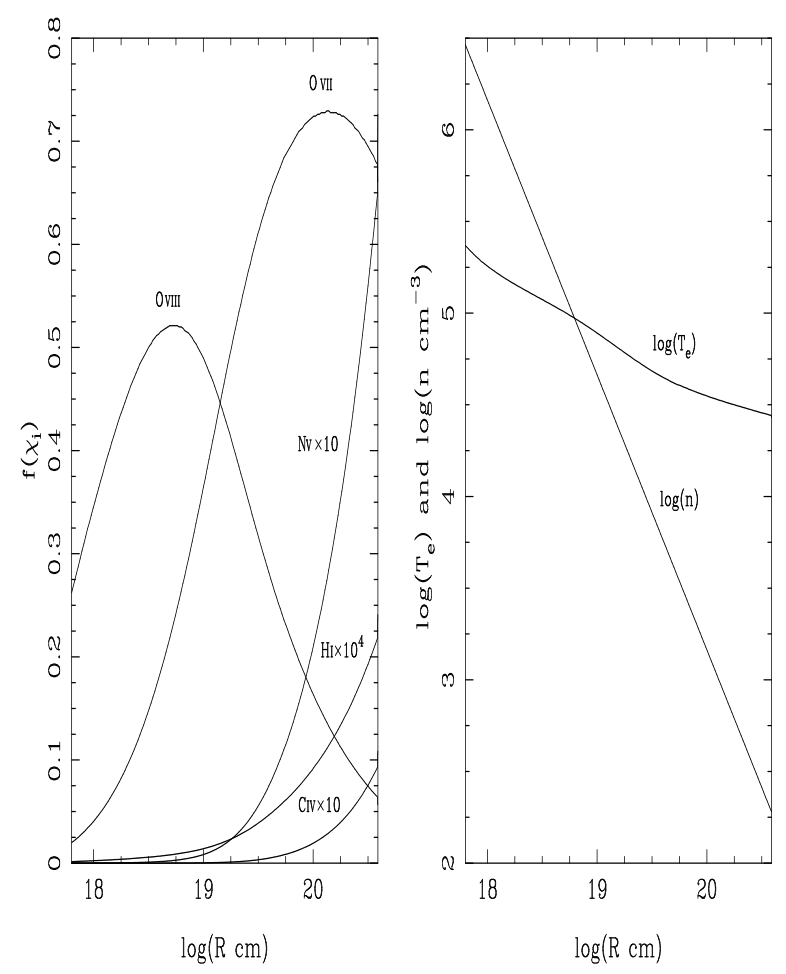

Fig. 4.- Left: Plot of the ion fraction as a function of radius for the continuously distributed model of NGC 5548. For clarity, C IV and N IV values have been multiplied by a factor of 10 . H I has been multiplied by a factor of $10^{4}$. Right: Logarithmic plot of the particle density $\left(\mathrm{cm}^{-3}\right)$ and temperature $\left({ }^{\circ} \mathrm{K}\right)$ as a function of radius $(\mathrm{cm})$ for the continuously distributed model of NGC 5548.

We first build a quasi-continuous model of MHD flow in NGC 5548 by smoothing the kinematic components along the line-of-sight, by imposing observational constraints on the total column density of 
hydrogen, and by retaining the $\propto R^{-3 / 2}$ density profile from the discrete component model. For simplicity, the outer edge of the continuous flow was set at $\log R \approx 20.6$ which coincides with the outer edge of the fifth component. Two free parameters, i.e., the volume filling factor and the inner radius, which we used as Cloudy input parameters, were tuned to fit observations of the column densities of $N(C \mathrm{IV})$, $N(N \mathrm{v}), N_{H}, N(O \mathrm{VII})$ and $N(O \mathrm{vIII})$. A reasonable fit to the ionic column densities is found for $\log \left(R_{1}\right) \approx 17.7$ and $\epsilon \sim 10^{-3}$ (Table 2). The first plot in Figure 4 clearly illustrates ionization stratification, with the ion fraction $\mathrm{O}$ VIII achieving a maximum at smaller radii, O VII at intermediate radii, and both $\mathrm{C}$ IV and $\mathrm{N} v$ peaking near the outer edge. The second plot in Figure 4 shows the density and electron temperature as a function of distance. Note the temperatures range from $\sim 1.6 \times 10^{4} \mathrm{~K}$ at larger radii to $\sim 2 \times 10^{5} \mathrm{~K}$ at smaller radii, where the bulk of the WA resides.

\subsection{Orientation Effects in AGNs and Soft X-Ray Absorption}

We now investigate the orientation effects in the continuous wind model of NGC 5548 by varying the viewer aspect angle. Figure 5 shows the column densities of $N(N$ v), $N(C$ IV $), N(H), N(O$ VII $)$ and $N(O$ as a function of viewer aspect angle $i$. The density profile along each aspect angle was adjusted so that the MHD flow is normalized to the solution for NGC 5548 (Table 2), which corresponds to the aspect angle $i=40^{\circ}$. Absorbing column densities seen by an observer with aspect angle $i>60^{\circ}$ are within the angular wedge shadowed by the BLR, i.e., by hydrogen column densities in excess of $10^{23} \mathrm{~cm}^{-2}$. Although we restrict our discussion to angles at $60^{\circ}$ or below, and to smaller column densities compatible with those in NGC 5548, larger column densities cannot be excluded for other objects. We adopt the generic model for NGC 5548 as a template, when applied to other Seyfert 1 galaxies.

Figure 5 shows that $N(O$ VII $)$ and $N(O$ VIII $)$ decrease with decreasing $i$, making detection of the oxy-

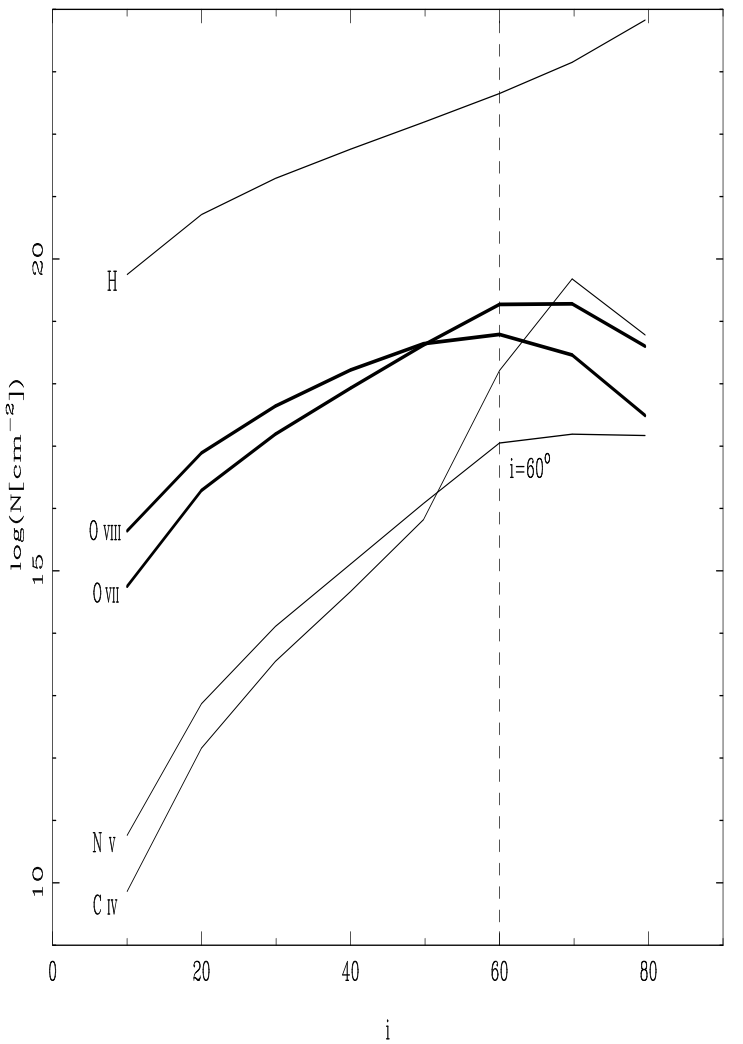

Fig. 5.- Plot of the $\log$ of the column densities $\left(\mathrm{cm}^{-2}\right)$ of $\mathrm{N} \mathrm{V}, \mathrm{C}$ IV, H, O VII and O VIII as a funcvitibh of observer aspect angle $i$ for the continuously distributed model. Values with angles greater than $60^{\circ}$ do not include contributions to the column from optically thick BLR clouds. The H column is therefore most likely to be an order of magnitude (or more) higher when $i>60^{\circ}$.

gen edges more difficult for small $i$. Using the instrument error bar in R97, and assuming a $2 \sigma$ detection criteria, absorption edges of $\mathrm{O}$ VII and $\mathrm{O}$ VIII will be lost for aspect angles less than $i \sim 30^{\circ}$ with the disk axis. Since no objects will be seen for $i>60^{\circ}$, due to obscuration, we restrict our analysis to angles $i<60^{\circ}$. In this case, if all Seyferts are taken as randomly oriented templates of NGC 5548, then about $\sim 73 \%$ of them will have detectable WA gas.

Figure 5 also shows that $N(O$ VII $)$ drops faster 
than $N(O$ VIII $)$ when viewed at smaller aspect angles, and drops below $N(O$ VIII $)$ for angles less than $50^{\circ}$. The fraction of Seyfert 1 galaxies with detectable WA showing $N(O$ VIII $)>N(O$ VII $)$ can be estimated from the ratio of the solid angle subtended on a sphere between the detection limit $30^{\circ}$ and $50^{\circ}$, and the solid angle between $30^{\circ}$ and $60^{\circ}$. This ratio is about $61 \%$, for current instrument sensitivity. For comparison, 8 out of 16 Seyfert 1 galaxies listed in R97 (50\%) have $N(O$ VIII $)>N(O$ VII $)$. (We used only Seyfert $1 \mathrm{~s}$ from R97 that have definite error bounds on both the tabulated values of optical depth $\tau_{O \text { VII }}$ and $\tau_{O \text { VIII }}$.) Finally, we note that the predicted range of $N_{H}$ varies from $\sim 10^{21} \mathrm{~cm}^{-2}$ to $\sim 10^{23} \mathrm{~cm}^{-2}$ within the observable range of aspect angles, and this compares favorably with the inferred range of $N_{H}$ in R97. Of course, one can question this approach, when one object is used as a template to describe the whole population of Seyferts, and the Seyfert sample in R97 is small. The model's consistency with the observations in these respects is nevertheless interesting. Taken at face value, the ratio of optical depths of $\mathrm{O}$ VII and $\mathrm{O}$ VIII ions can serve as a new diagnostic for AGN aspect angles.

\subsection{Diffuse Emission from the UVX Absorber}

The UVX absorbing gas reprocesses and scatters the incident continuum into diffuse emission across the UV and soft X-rays. We used our "generic" UVX absorber for NGC 5548 to estimate the magnitude of this emission. We computed Cloudy models for thermal local line widths and one, in which microturbulence broadened the local line width $\left(\sigma_{\text {turb }}=\right.$ $100 \mathrm{~km} \mathrm{~s}^{-1}$ ). In the latter scenario, optically thick lines are desaturated, and the cross-section for photon pumping of the line's upper level (continuum resonance line scattering) is elevated, enhancing the line intensities. Photon pumping may be especially important for the soft X-ray lines because of their small Boltzmann factors in photoionized gas. However, even under the assumption that the observer can see the gas represented by our generic UVX absorber distributed over $4 \pi$ steradians over the continuum source, the diffuse emission is relatively small. This is expected, given the reported small optical depths of the absorber in this object. We find that this gas contributes at most $\sim 20 \%$ to the observed UV narrow line fluxes of Ly $\alpha, \mathrm{C}$ IV, and N V in NGC 5548 (Goad \& Koratkar 1998). Emission of this significance may be sufficient to partially fill in some of the absorption troughs. Depending upon the covering fraction and the contribution from photon pumping the diffuse soft X-ray line emission could be also be significant in comparison to the absorption edge depths (Netzer 1993). We will have to await the results from the upcoming missions of Chandra, XMM, and Constellation- $X$ to get hard observational constraints from this portion of the spectrum.

\section{SUMMARY}

We have applied the EBS hydromagnetic wind model from a clumpy molecular accretion disk to the problem of UVX-ray absorption systems observed in Seyfert galaxies. We have studied the state of the UVX absorbing gas, with a particular emphasis on NGC 5548, where five discrete UV absorption systems seen in this object were modeled. Extending our previous work on the dynamics of the BLR gas in NGC 5548 (Paper 1), we first inferred the location of each component relative to the continuum source. By estimating the ratio of the ion fractions of $\mathrm{N} \mathrm{V}$ to $\mathrm{C}$ IV from the observed column densities and the use of photoionization modeling, we have determined possible values for the density, electron temperature and the ion fractions of $\mathrm{C}$ IV, $\mathrm{N} \mathrm{v}, \mathrm{O}$ VI, O VII O VIII, $\mathrm{H}$ and $\mathrm{H}$ I. Optimization over the set of possible values yielded the ion column densities in each kinematic component and allowed us to test the radial and polar density distributions of the WA in NGC 5548, within the EBS framework. We have also estimated the size and volume filling factor of each component, using the FWHMs and electron temperatures, and found that the flow in each component is clumpy. Xray absorption by $\mathrm{O}$ VII and $\mathrm{O}$ VIII column densities seen in NGC 5548 have been accounted for in the five UV absorption components, though main contribution to the X-ray absorption comes from two compo- 
nents. We, therefore, were able to explain the UVX absorption columns within the framework of the same dynamical model. An additional important point is that the model parameters used here are the best fitting parameters from Paper 1 to explain the broad emission-line variability in NGC 5548 during the 1989 and 1993 observing campaigns.

Secondly, we find that the WA gas in NGC 5548 lies at larger radii from the central source than the BLR gas and at larger altitudes above the disk. This means that WA exists in the outer parts of the hydromagnetic disk wind, and as such is not a continuation of the BLR flow. As a result we do not expect it to contribute to absorption along the line of sight unless the aspect angle is near the obscuring torus. With regard to the spatial extent of the WA gas we find it extends in both radial and polar directions from the central continuum source, and is ionization stratified.

Thirdly, we have modeled the WA in NGC 5548 also as a continuous flow, in order to investigate the generic properties of the UVX absorption outflows in Seyfert galaxies. We find that orientation effects may fully account for the fraction of objects with detectable WAs. Furthermore, the model predicts that the ratio of optical depths of O VIII to O VII can serve as a new diagnostic of orientation of an AGN, namely of its rotation axis - an issue of a particular interest in AGN theory. In addition, we find that the UV line emission from the UVX flow probably accounts for no more than $\sim 20 \%$ of the NLR emission in lines, such as Ly $\alpha, \mathrm{C}$ IV, N v, and O VI, though diffuse emission in the soft X-rays may be significant under some conditions.

A thermal stability analysis has been carried out for the model. We find that all of the five components in NGC 5548 are stable, though two components (1 and 3) lie near an unstable, to isobaric perturbations, region on the S-curve. By considering the effects of magnetic field on the thermal stability of the WA, we find that these components can be further thermally stabilized by a modest field, even in the presence of substantial continuum fluctuations, even when considering extremes in SED or metallicity. Such stabilization by magnetic fields may explain the ubiquity of WA gas in AGNs.

As more objects are observed with more sensitive instruments (e.g., Chandra and XMM coupled with the $H S T$ ), better statistical tests of the wind model can be carried out, involving determination of the fraction of AGNs with detectable WA gas, the frequency of objects in which the $\mathrm{O}$ VII column density is larger than the $\mathrm{O}$ VIII column density, and a determination of the range of $N_{H}$. Future higher resolution X-ray spectra $(A S C A, X M M)$ will provide additional kinematic information, such as oxygen edge and resonance line absorption velocities and help to verify the link between X-ray and UV absorption.

We gratefully acknowledge illuminating discussions with Roger Blandford, Mike Crenshaw, Arieh Königl, Richard Mushotzky and Chris Reynolds. We thank Gary Ferland for the use of Cloudy. This work was supported in part by NASA grants NAG5-3841, WKU-522762-98-06, WKU-521782-99-04 and HST AR07982.01-96A.

\section{A. THERMAL STABILITY OF WARM ABSORBING GAS}

The WA gas is found in a wide range of densities, temperatures, ionization and metallicity. It seems plausible, that this ubiquity of WAs in AGNs is related to their thermal stability. Permeating magnetic fields which are expected to play an important dynamical role in AGNs, can affect the thermal stability of the absorbing gas. Therefore, along with Field (1965) and EBS, we discuss the general aspects of thermal stability of magnetized gas and its application to NGC 5548 and to a generic model of the WA, subject to AGN radiation field.

\section{A.1. The "S-curve" and Thermal Stability: General Considerations}

For a gas in thermal equilibrium, the heating rate per unit volume, $G(n, T)$, is balanced by the cooling rate per unit volume, $\Lambda(n, T)$. If $H$ is the net heating 

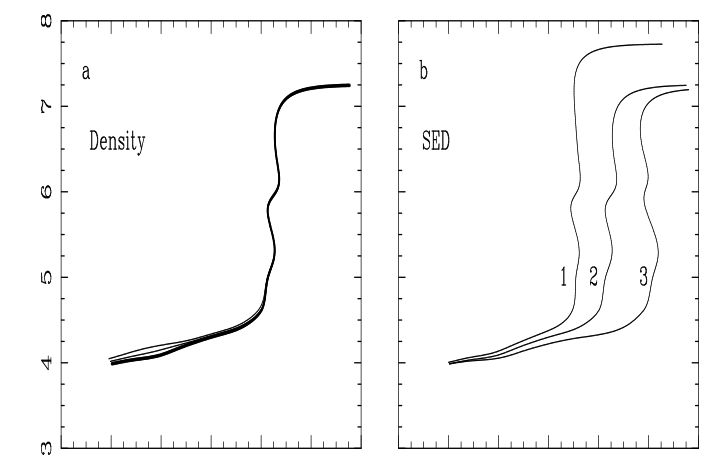

$\hat{H}$
bo
0
+
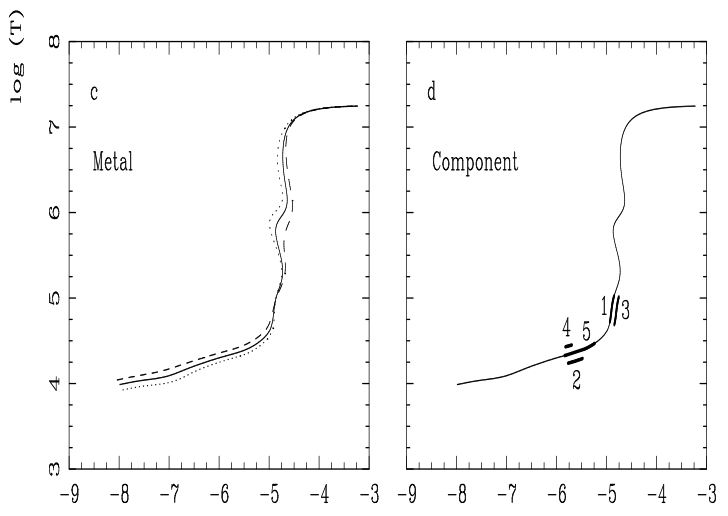

$\log (\mathrm{U} / \mathrm{T})$

Fig. 6.- S-curves for various parameters. (a) Scurves for the SED of NGC 5548 for densities $10^{5}$, $10^{6}, 10^{7}$ and $10^{8} \mathrm{~cm}^{-3}$. The thick line contains both the $10^{5} \mathrm{~cm}^{-3}$ and $10^{6} \mathrm{~cm}^{-3}$ S-curves. Note that above $10^{5} \mathrm{~K}$ all the S-curves merge, illustrating that the S-curve is relatively insensitive to differences in density. (b) S-curves corresponding to different incident continuum SEDs. The S-curve labeled 1 is for the SED of MCG 6-30-15; the S-curve labeled 2 is for the SED of NGC 5548; the S-curve labeled 3 is for the Mathews \& Ferland (1987) composite spectrum of quasars. The hardness of the SED decreases from left to right. (c) S-curves for the SED of NGC 5548 with three different metal abundances. The solid line corresponds to solar abundances; the dashed line is for a metal abundance of $0.5 \times$ solar; the dotted line is for an abundance of $2.0 \times$ solar. (d) Location of the 5 discrete absorption components observed by C99 on the S-curve for NGC 5548. Segments are offset for clarity. Components 1 and 3 lie close to an unstable (to isobaric perturbations) portion of the S-curve. rate defined by

$$
H \equiv G(n, T)-\Lambda(n, T),
$$

then equilibrium occurs when $H=0$. If gas is slightly perturbed, i.e., $H \neq 0$, the gas will either heat up or cool down. The change in the gas temperature defines whether it is thermally stable (Field 1965; Krolik, McKee \& Tarter 1981). The equation $H=0$ is more conveniently treated when mapped to the $\log T-\log U / T$ plane where the equilibrium curve has roughly an S-shape. Thermal stability of gas depends on the details of the S-curve, and the path that a perturbation is allowed to move along. Figure 6a displays a series of S-curves corresponding to different hydrogen gas densities for the SED of NGC 5548. The curves are relatively insensitive to the gas densities considered and become virtually indistinguishable above $T \approx 10^{5} \mathrm{~K}$.

To analyze thermal stability of gas, one must be able to follow the path of the perturbed gas relative to the S-curve. Figure 7 shows a small segment of the S-curve for NGC 5548, having a negative slope in the vicinity of point $\mathrm{P}$. To the right of the S-curve, the net heating $H$ is positive, i.e., perturbed gas always heats up. To the left of the S-curve, the net heating $H$ is negative, meaning that perturbed gas always cools down. Crossing the S-curve at the equilibrium point $P$ are three paths (G, L and I), representing different alternatives for an evolving perturbation off the point P. Path I, a vertical line, represents an isobaric perturbation? $\mathrm{G}$ and $\mathrm{L}$ represent perturbations constrained to paths with slopes greater (less negative) and with slope less (more negative) than the slope of the S-curve at P, respectively. Along I and L, a small perturbation off the S-curve leads to a dramatic change in temperature. Perturbations constrained to move along $\mathrm{G}$, on the other hand, are thermally stable since they are driven back to P. Hence, the thermal stability of the gas depends on the slope of developing perturbation in $\log T-\log U / T$ plane. The gas is thermally stable when this slope is greater (less negative) than the slope of the S-curve at P. For an ionized

\footnotetext{
${ }^{2}$ If luminosity and radius are fixed then $U / T \propto 1 / n T$, therefore, $U / T=$ constant implies $n T$ is constant. Hence, the gas moving on vertical lines in the $T$ vs. $U / T$ plane evolves isobarically.
} 


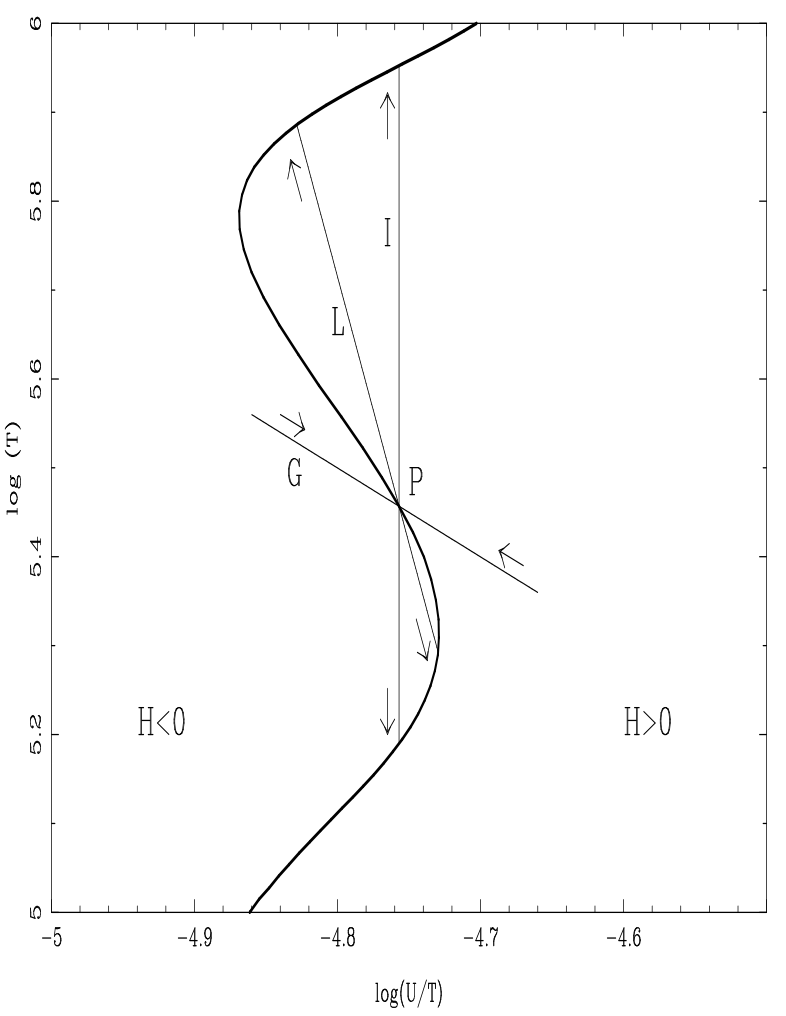

Fig. 7.- Schematic depiction of an unstable (to isobaric perturbations) portion of the S-curve. To the left of the S-curve cooling exceeds heating. To the right of the S-curve heating exceeds cooling. Different perturbation constraints (paths G, L, and I) are shown crossing the point P. Arrows show the direction gas will evolve along a constraint when perturbed off the point $P$. Path I corresponds to a thermally unstable isobaric perturbation at P. Path L has a slope less than the S-curve at point $\mathrm{P}$, therefore, perturbations constrained on this path are unstable. Path $G$ has a slope greater than the $\mathrm{S}$-curve at $\mathrm{P}$, so perturbations constrained to this path are stable.

gas permeated by a magnetic field, as shown below, perturbations move along inclined paths in this diagram, e.g., as G or L in Figure 7. Since stability depends on the relative steepness of the S-curve, as compared to that of the the perturbation path, we first investigate the factors which influence the shape and, therefore, the slope of the S-curve.

\section{A.2. Factors Affecting the S-curve: SED and Metallicity}

The overall shape of the S-curve is affected by the SED. Figure 6b, shows the S-curves for three different SEDs with labels corresponding to SED with photon index $\Gamma=1.8$ and energy cutoffs between $13.6 \mathrm{eV}$ and $40 \mathrm{keV}$ (this SED was utilized in Reynolds 1995), to the SED used in our modeling of NGC 5548, and to the typical quasar SED deduced by Matthews \& Ferland (1987). The sequence is in terms of decreasing hardness of the continuum and the greatest effect is on the upper branch of the S-curve. Here the temperature is mainly controlled by Compton heating and cooling. In the limit of large $\log (U / T)$, the S-curve is asymptotic to the Compton temperature $T_{C}$ which is given by $T_{C}=h\langle\nu\rangle / 4 k$, where $\langle\nu\rangle$ is the flux-averaged photon frequency. Higher $\langle\nu\rangle$ corresponds to harder SED, and, therefore, higher $T_{C}$, explaining the sequence of decreasing upper branch temperatures.

The horizontal separation between S-curves (Fig. 6b) is due to decrease in the continuum hardness, when the percentage of high energy photons $(h \nu>1$ Ryd) that heat the gas per unit frequency interval decreases. Decreasing the hardness, allows the gas to cool down, unless compensated for by increasing the number of high energy photons, which is the same as increasing the ionization parameter. Thus, for a given value of $T$, the S-curve lies further to the right (i.e., it has higher $U / T$ values which correspond to higher values of $U)$ as the hardness decreases. In spite of a wide range of hardness, the slopes of the S-curves vary little, particularly in regions where the slope is negative. We further note that in all cases considered here, the $\mathrm{S}$-curve slopes is always less than -1 , in regions where the slope is negative. This is an important point because, as we show below, the path that gas perturbation treaded by a magnetic field follows, has slope close to -1 , resulting in thermal stabilization.

The bumps and wiggles in an S-curve are due to the metallicity of the gas. If AGNs are fueled with galactic ISM, its composition may reflect intense localized star formation which is typically concentrated within nuclear and circumnuclear regions and, there- 
fore, uniformity of composition is not guaranteed. In addition, when grains are directly exposed to an intense central continuum, their mantles will be destroyed and only the graphite cores will be able to survive photo-desorption (Draine \& Salpeter 1979) at distances and in the radiation field relevant for WA in NGC 5548. This introduces the possibility of metallicity gradients within the WA environment. Gas abundances are important, because at WA temperatures line radiation by heavier elements, such as oxygen, constitute the dominant coolant. Thus metallicity can influence the thermal structure and stability of the WA gas. This is demonstrated below.

Figure 6c shows S-curves for the SED of NGC 5548 for gas with three different metallicities. At temperatures above $T \approx 10^{5} \mathrm{~K}$, higher metallicity curves are shifted further to the left than lower metallicity curves in the $\log T$ vs. $\log U / T$ plot. This is because the presence of more metal ions gives the gas a net higher energy absorption cross section than that at a lower metallicity, so that a particular temperature is achieved with a lower ionization parameter. In addition, extra electrons provided by metal enhancement are able to recombine with $\mathrm{H}^{+}$(and $\mathrm{He}^{++}$), thus the effective ionization of the gas is lower for a given ionization parameter. For example, along a line of $\log T \approx 5.85$, the ionization parameter increases from roughly 0.85 to 1.14 to 1.25 , as the metallicity drops. Below $T \approx 10^{5} \mathrm{~K}$, the order is reversed because the metal ions recombine and become more efficient coolers via line emission than heaters via absorption. Inspection of the Figure $6 \mathrm{c}$ reveals that the slopes of the S-curves for different metallicities are similar, and in the regions where the slopes are negative, they all are less than -1 . So, metallicity differences, even as large as a factor of 2 greater or less than solar, will not affect our conclusions about thermal stability of magnetically-dominated gas.

\section{A.3. Thermal Stability of Magnetized Gas}

For isobaric perturbations, there are no dynamical effects and the resulting evolution of the perturbation is controlled by thermal effects only. In the absence of a magnetic field, an isobaric path corresponds to a vertical line (path I in Figure 7). For this kind of perturbation, regions where the slope of the S-curve is negative are thermally unstable. The location of the discrete UV/X-ray WA model is shown in the $\log T$ vs. $\log U / T$ plane (Fig. $6 \mathrm{~d}$ ). The temperature range of absorption components 1 and 3 lies close to a region which is thermally unstable to isobaric perturbations, so changes in continuum luminosity may drive these components into an unstable region. 3 Components 2, 4 and 5 are in a region where the S-curve slope is positive, so these components are thermally stable.

The mere existence of kinematic components 1 and 3 in NGC 5548 hints that the gas is thermally stable, despite being close to unstable region. Such a stabilization mechanism can be provided by magnetic fields permeating the gas (Field 1965; EBS). In the presence of magnetic field, isobaric perturbations are constrained to evolve along the curve defined by $n k T+B^{2} / 8 \pi=A$ where $A$ is a constant. To analyze thermal stability, we rewrite this expression in terms of $T$ and $\frac{U}{T}$ and show that when plotted in the $\log T$ vs. $\log U / T$ plane, the slope is approximately equal to -1 . Assuming magnetic flux freezing, the magnetic field scales with the gas density $B^{2}=\phi^{2} n^{2}$, where $\phi$ is a constant. Substitution into the expression for total pressure gives $n k T+\phi^{2} n^{2} / 8 \pi=A$. We eliminate $n$ by solving

$$
\frac{U}{T}=\frac{L_{i o n}}{<E_{i o n}>4 \pi R^{2} c n T}
$$

for $n$. This gives

$$
n=\frac{E}{\left(\frac{U}{T}\right) T},
$$

where $E=L_{i o n} /<E_{i o n}>4 \pi R^{2} c$. Substitution into the expression for total pressure gives

$$
\frac{E k}{\frac{U}{T}}+\frac{\phi^{2} E^{2}}{8 \pi T^{2}\left(\frac{U}{T}\right)^{2}}=A,
$$

\footnotetext{
${ }^{3}$ The temperature uncertainty for each component is estimated by searching for the largest and smallest values temperature values in the $(n, N(C$ IV $))$ solution grid that are adjacent to the optimal solution.
} 
and solving for $T$ yields

$$
T=\frac{\frac{\phi E}{\sqrt{8 \pi}}}{\sqrt{A\left(\frac{U}{T}\right)^{2}-E k\left(\frac{U}{T}\right)}} .
$$

To thermally stabilize the gas requires

$$
\frac{d \log (T)}{d \log (U / T)}>S,
$$

where $S$, the slope of the S-curve in the $\log T$ vs. $\log U / T$ plane, and the derivative on the left hand side of Equation A6 are evaluated at the point where Equation A5 crosses the S-curve, yielding

$$
\frac{d \log T}{d \log \left(\frac{U}{T}\right)}=-1-\frac{1}{2} \beta
$$

where $\beta$ is the ratio of gas to magnetic field pressures. Note, that when the magnetic field $B \rightarrow 0$, then $\beta \rightarrow \infty$, and $d \log T / d \log \left(\frac{U}{T}\right) \rightarrow-\infty$, recovering the strictly isobaric case. At the other extreme lies the limiting case of the cold EBS flow, in which the magnetic pressure completely dominates the gas pressure $(\beta<<1)$, so $d \log T / d \log \left(\frac{U}{T}\right) \approx-1$.

The minimum value of $B$ required to stabilize the gas is found by equating the slope of the S-curve to Equation $\mathrm{A} 7$, and solving for $\beta_{\text {min }}$. Figure 8 shows $1 / \beta_{\min }$ values as a function of temperature for the two regions of instability, for the SED of NGC 5548. For stable regions, no magnetic field is needed, so $1 / \beta_{\min } \rightarrow 0$. Components 1 and 3 are close to the thermally unstable temperature corresponding to the left peak in Figure 8 . The maximum $1 / \beta_{\text {min }}$ occurs at $\log T=5.55$, where $1 / \beta_{\text {min }}=0.395$, giving

$$
\frac{1}{\beta}=\frac{\frac{B^{2}}{8 \pi}}{n k T}=0.395
$$

where $B$ and $n$ are the values for $\log T=5.55$. Thus, according to Equation A8, if the value of the magnetic pressure is about $40 \%$ of the gas pressure or larger the gas is stabilized. Cloudy calculations and our wind model yield a density of $\log n=4.99$ at the center of component 1 . With this density a lower bound on the magnetic field required for stabilization can be estimated. Solving Equation A8 for $B$ gives

$$
B=\sqrt{8 \pi \times 0.395 n k T} .
$$

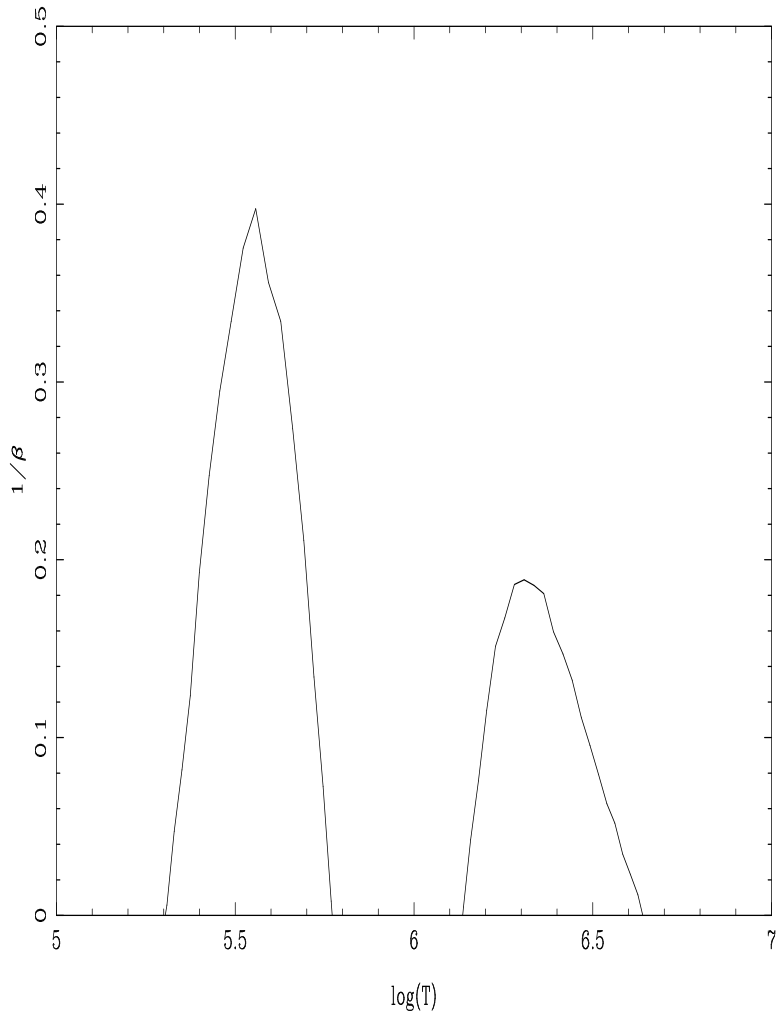

Fig. 8. - $1 / \beta$ vs. $T$ for the S-curve of NGC 5548. $\beta$ is the minimum value required to stabilize gas at the corresponding temperature.

In the regime of the left peak, the gas is stable at all possible temperatures, if $B \gtrsim 7.0 \times 10^{-3}$ Gauss. It should be noted, however, that the bound on $B$ is determined only from the stability requirement. A more stringent requirement from the EBS model is that the flow is a "cold" MHD flow, which means $1 / \beta>>1$, superseding the above criteria. The implication is that the slope given by Equation A7 is nearly equal to -1 . We have noted above that variations in SED or metallicity do not significantly change the slopes of the negative parts of the S-curve, and that the slopes are always less than -1 , corresponding to case $\mathrm{G}$ of Figure 7, in which the slope of the perturbation is larger than the slope of the S-curve. Thus, in the case of NGC 5548, as described by the EBS flow, regions of the S-curve that correspond to thermally unstable regions to isobaric perturbations are thermally stabilized by the presence of a magnetic field. 


\section{REFERENCES}

Arav, N., Shlosman, I., Weymann, R.J. (Eds.) 1997, ASP Conference Series, Vol. 128, Mass Ejection from Active Galactic Nuclei

Blandford, R.D. 1990, Saas-Fee Advanced Course, Vol. 20, Active Galactic Nuclei, ed. Courvoisier, T.J.-L., Mayor, M. Springer-Verlag, 161

Blandford, R.D. \& Payne, 1982, MNRAS, 199, 883

Bottorff, M.C., Korista, K.T., Shlosman, I., Blandford, R.D. 1997, ApJ, 479, 200 (Paper 1)

Brandt, W.N., Laor, A., \& Wills, B.J. 1999, ApJ, in press

Bromage, G.E., et al. 1985, MNRAS, 215, 1

Clavel, J. et al. 1991, ApJ, 366, 64

Crenshaw, M.D., Kraemer, S.B., Boggess A., Maran, S.P., Mushotzky, R.F., Wu, C. 1999, ApJ, 516, 750 (C99)

Crenshaw, M.D., Kraemer, S.B. 1999, ApJ, 521, 572

de Kool, M., Begelman, M.C., 1995, ApJ, 445, 448

Draine, B.T., Salpeter, E.E. 1979, ApJ, 231, 438

Emmering, R.T., Blandford, R.D., Shlosman, I. 1992, ApJ, 385, 460

Fabian, A.C., et al. 1994, PASJ, 46, L59

Ferland, G.J. 1996, Hazy, a Brief Introduction to Cloudy, University of Kentucky Department of Physics and Astronomy Internal Report

Field, G.B. 1965, ApJ, 142, 531

Galeev, A.A., Rosner, R., Vaiana, G.S. 1979, ApJ, 229,318

Gallagher, S.C., Brandt, W.N., Sambruna, R.M., Mathuџ,rich, M-H., Comastri, A., Komossa, S., Crane, P. S., \& Yamasaki, N. 1999, ApJ, 519, 549

George, I.M., Turner, T.J., R.A., Netzer, H, Nandra, K., Mushotzky, R.F., Yaqoob, T. 1998, ApJS, 114,73

Green, P.J., Mathur, S.1996, ApJ, 462, 637

Goad, M.R., \& Koratkar, A.P. 1998, ApJ, 495, 718

Komossa, S., Fink, H. 1997, A\&A, 327, 483

Königl, A., Kartje, J.F. 1994, ApJ, 434, 446

Korista, K.T., et al. 1995, ApJS, 97, 285

Koratkar, A., et al. 1996, ApJ, 470, 378

Kriss, G.A., et al. 1992, ApJ, 392, 485
Krolik, J.H., Kriss, G.A. 1995, ApJ, 447, 512

Krolik, J.H., McKee, C.F., Tarter, B.C. 1981, ApJ, 249,422

Leech, K.J., Penston, M.V., Snijders, M.A. 1991, MNRAS, 249, 24p

Mathur, S., \& Elvis, M. 1995, ApJ, 455, L9

Mathur, S., Elvis, M., \& Singh, K.P. 1995, ApJ455, L9

Mathur, S., Elvis, M., Wilkes, B. 1995, ApJ, 452, 230

Mathur, S., Elvis, M., Wilkes, B., 1999, ApJ, 519, 605 (MEW99)

Mathur, S., Wilkes, B., Aldcroft, T. 1997, ApJ, 478, 182

Mathur, S., Wilkes, B., Elvis, M. 1999, ApJ, in press

Mushotzky, R.F., 1997, ASP Conference Series, Vol. 128, Mass Ejection from Active Galactic Nuclei, ed. Arav, N., Shlosman, I., Weymann, R.J. (ASP), 141

Netzer, H. 1993, ApJ, 411, 594

Nicastro, F., Fiore, F., Preola, G.C., Elvis, N. 1998, astro-ph/9808316

Otani, C., et al. 1996, PASJ, 48, 211

Reynolds, C.S., Fabian, A.C. 1995, MNRAS, 273, 1167

Reynolds, C.S. 1997, MNRAS, 286, 513

Shields, J.C., \& Hamann, F. 1997, ApJ, 481, 752

Shull, M.J.,\& Sachs, E.R. 1993, ApJ, 416, 536

Siebert, J., Matsuoka, M., Brinkmann, W., Cappi, M., Mihara, T. \& Takahashi, T. 1996, A\&A, 307,8 1999, astro-ph/9907150, A\&A, in press

Verón, P., Verón-Cetty M.-P., Tarenghi, M. 1985, A\&A, 150, 317

Voit, M.G., Shull, M., Begelman, M.C. 1987, ApJ, 316,573

Walter, R., Ulrich, M.-H., Courvoisier, T.J.-L., Buson, L.M. 1990, A\&A, 233, 53

Walter, R., Orr, A., Courvoisier, T.J.-L., Fink, H.H., Makino, F., Otani, C., \& Wamsteker, W. 1994, A\&A, 285, 119 
Weymann, R.J. 1997, ASP Conference Series, Vol. 128, Mass Ejection from Active Galactic Nuclei, ed.

Arav, N., Shlosman, I., Weymann, R.J., 3 
Table 1a

$A S C A$ R97 X-ray Observational Summary NGC 5548

(Adopted Target Values ${ }^{a}$ )

\begin{tabular}{ll}
\hline \hline $\log \left(N_{H}\right)$ & 21.71 \\
$\log \left(N_{O \text { VII }}\right)$ & 17.95 \\
$\log \left(N_{O \text { VIII }}\right)$ & 18.20 \\
\hline
\end{tabular}

Table $1 \mathrm{~b}$

HST C99 UV Observational Summary NGC 5548 (Adopted Target Values ${ }^{a}$ )

\begin{tabular}{lllll}
\hline \hline comp. & $\log [N(N \mathrm{~V})]$ & $\log [N(C \mathrm{IV})]$ & $\log \left[N\left(H_{\mathrm{I}}\right)\right]$ & $\log \left[\frac{f(N \mathrm{~V})}{f\left(C_{\mathrm{IV}}\right)}\right]$ \\
\hline 1 & $14.30<$ & $13.04<$ & $14.13^{b}$ & $2.14>$ \\
2 & 13.78 & 13.45 & 13.88 & 0.91 \\
3 & 14.59 & 13.68 & 14.25 & 1.49 \\
4 & 14.81 & 14.46 & 14.52 & 0.93 \\
5 & 14.04 & 13.61 & 13.11 & 1.01 \\
\cline { 1 - 3 }$\Sigma$ & 15.17 & 14.62 & 14.87 &
\end{tabular}

a All values have assigned error bars of $\pm \log (2.0)$ except for those with a $<$, which indicates a lower bound or $>$, which indicates an upper bound.

${ }^{b}$ STIS observation (Crenshaw \& Kraemer 1999) 
Table 2

Model Solution for the Continuously Distributed Warm Absorber in NGC 5548

\begin{tabular}{ll}
\hline \hline parameter & $\log (\text { value })^{a}$ \\
\hline $\mathrm{R}_{\min }$ & 17.75 \\
$\mathrm{R}_{\max }$ & 20.59 \\
$\mathrm{n}_{\min }$ & 6.455 \\
$\mathrm{n}_{\max }$ & 2.253 \\
$\mathrm{~N}(\mathrm{H})$ & 21.76 \\
$\mathrm{~N}(\mathrm{H} \mathrm{I})$ & 15.94 \\
$\mathrm{~N}(\mathrm{C}$ IV $)$ & 14.70 \\
$\mathrm{~N}(\mathrm{~N}$ V $)$ & 15.13 \\
$\mathrm{~N}(\mathrm{O}$ vII $)$ & 17.94 \\
$\mathrm{~N}(\mathrm{O}$ VIII $)$ & 18.22 \\
$\epsilon$ & -2.716 \\
\hline
\end{tabular}

${ }^{a}$ values in cgs units. 
Table 3a

Optimal EBS Solution Column Densities ${ }^{a}$

\begin{tabular}{cccccccc}
\hline \hline comp. & C IV & N v & O VI & O VII & O VIII & H & H I \\
\hline 1 & 13.50 & 14.30 & 16.15 & 17.73 & 17.84 & 21.29 & 15.57 \\
2 & 13.45 & 13.48 & 14.57 & 14.51 & 13.00 & 18.14 & 14.11 \\
3 & 13.62 & 14.41 & 16.25 & 17.80 & 17.88 & 21.33 & 15.66 \\
4 & 14.46 & 14.51 & 15.65 & 15.65 & 14.20 & 19.22 & 15.14 \\
5 & 13.49 & 13.74 & 15.11 & 15.56 & 14.55 & 18.88 & 14.35 \\
\hline$\Sigma$ & 14.62 & 14.94 & 16.58 & 18.07 & 18.16 & 21.61 & 16.00 \\
\hline
\end{tabular}

${ }^{a}$ Logarithims of column densities in units of $\mathrm{cm}^{-2}$

Table 3b

Optimal EBS Solution Physical Parameters ${ }^{a}$

\begin{tabular}{cccccccccccc}
\hline \hline comp. & $\mathrm{v}_{\mathrm{obs}}^{\mathrm{b}}$ & $\mathrm{FWHM}^{b}$ & $\mathrm{v}_{\mathrm{s}}$ & $\Delta \mathrm{v}$ & $\frac{f(N \mathrm{v})}{f(C \text { IV })}$ & $\mathrm{R}$ & $\Delta \mathrm{R}$ & $\mathrm{n}$ & $\mathrm{T}$ & $\mathrm{U}$ & $\epsilon$ \\
\hline 1 & -1060 & 114 & 9.8 & 104.2 & 1.39 & 18.8 & 18.1 & 5.0 & 4.87 & -0.03 & -1.8 \\
2 & -655 & 45 & 5.4 & 39.5 & 0.61 & 19.2 & 18.3 & 5.4 & 4.37 & -1.29 & -5.6 \\
3 & -518 & 90 & 9.6 & 80.4 & 1.37 & 19.4 & 18.9 & 3.8 & 4.86 & -0.05 & -1.4 \\
4 & -344 & 156 & 5.4 & 150.6 & 0.63 & 19.8 & 19.8 & 4.2 & 4.36 & -1.23 & -4.8 \\
5 & -165 & 74 & 5.9 & 68.7 & 0.83 & 20.4 & 20.4 & 2.6 & 4.44 & -0.85 & -4.1 \\
\hline
\end{tabular}

${ }^{a}$ Velocities are given in $\mathrm{km} \mathrm{s}^{-1}$. All other entries are given as logarithm of the value expressed in cgs.

${ }^{b}$ Values are from C99. 
Table 4

Component Ion Recombination Times and Percentage Total Column Contributions for NGC 5548

\begin{tabular}{ccccccc}
\hline \hline $\mathrm{n}$ & $\mathrm{C} \mathrm{IV}^{a}$ & $\%^{b}$ & O VII & $\%$ & O VIII & $\%$ \\
\hline 1 & 2.71 & 7.59 & 5.39 & 45.71 & 6.62 & 47.86 \\
2 & 4.23 & 6.76 & 5.46 & 0.03 & 5.54 & $>0.01$ \\
3 & 3.96 & 10.00 & 6.60 & 53.70 & 7.80 & 52.48 \\
4 & 5.30 & 69.18 & 6.59 & 0.38 & 6.57 & 0.01 \\
5 & 6.39 & 7.41 & 8.14 & 0.31 & 8.30 & 0.02 \\
\hline$\Sigma^{c}$ & 5.51 & & 6.43 & & 7.54 & \\
\hline
\end{tabular}

${ }^{a}$ Values are the $\log t(s)$ of the absolute value of the mean component recombination time. All C IV responses are anticorrelated and all O VII and O VIII responses are correlated with a continuum drop.

${ }^{b}$ Percentage of the total ionic column density.

${ }^{c}$ Values are the $\log t(s)$ of the absolute value of the mean recombination time averaged over all components and weighted by their relative column density contributions. 